\title{
Development of intein-inspired amide cleavage chemical device
}

Chiaki Komiya $^{\dagger}$, Keisuke Aihara ${ }^{\dagger}$, Ko Morishita, Hao Ding, Tsubasa Inokuma, Akira Shigenaga, Akira Otaka*

${ }^{\dagger}$ Contributed equally

Institute of Biomedical Sciences and Graduate School of Pharmaceutical Sciences, Tokushima University Shomachi, Tokushima 770-8505, Japan

E-mail:

aotaka@ph.tokushima-u.ac.jp

\section{SUPPORTING INFORMATION}

\section{Content:}

Copies of NMR spectra for all compounds 
2-Nitrobenzyl $\{2-[($ tert-butoxycarbonyl)amino]ethyl $\}$ ethylcarbamate $\mathbf{3}$
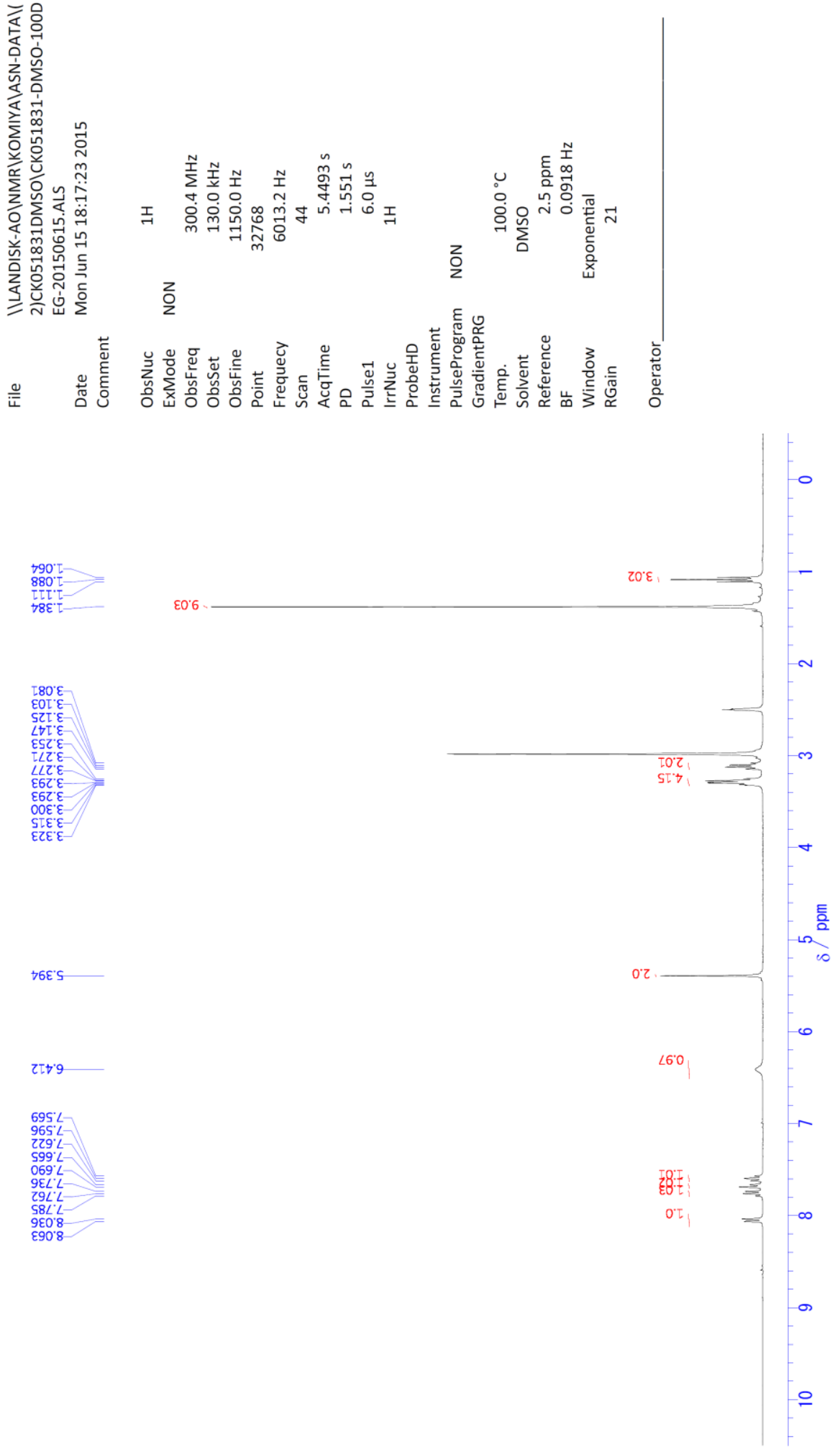

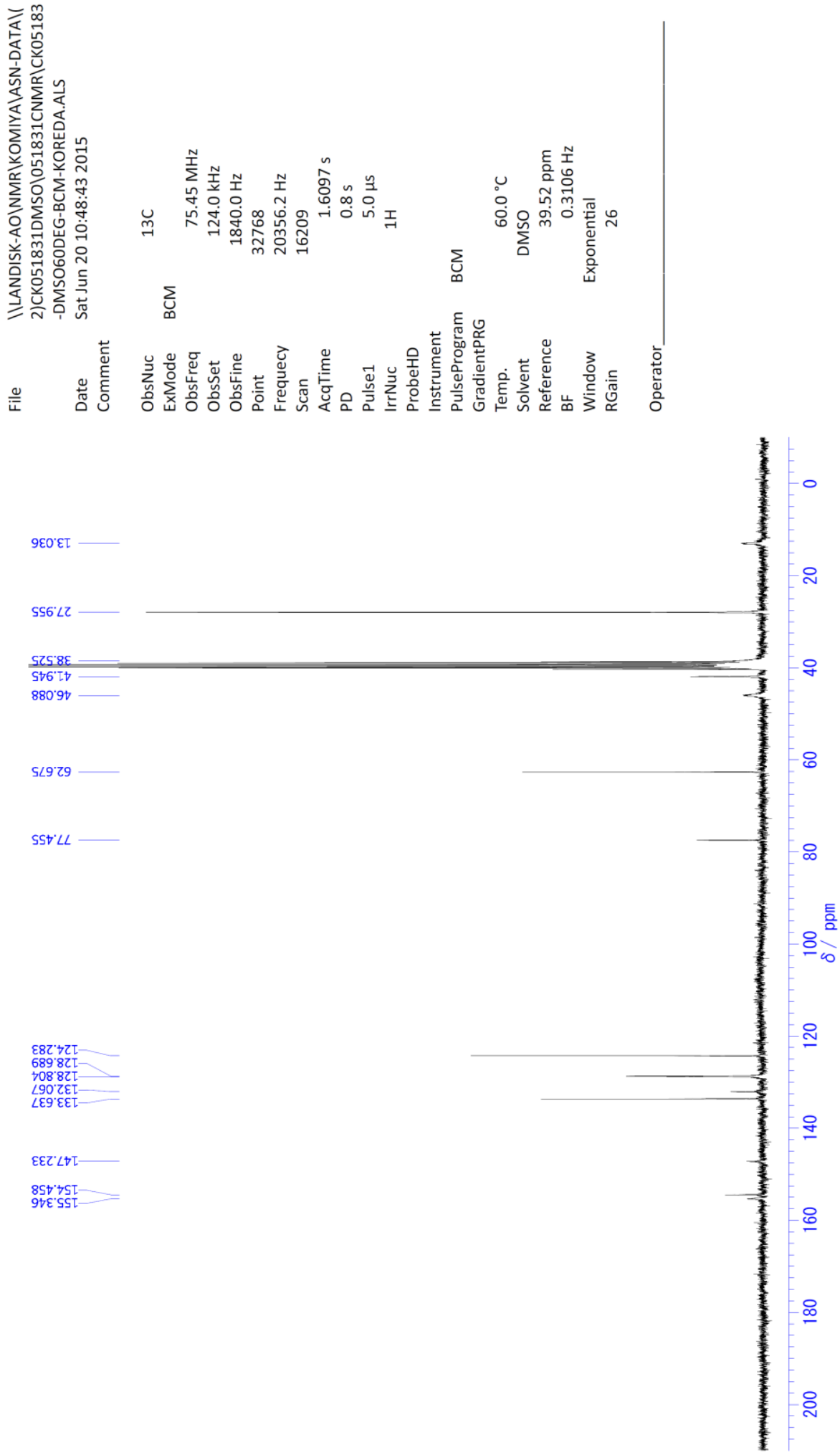
(S)-3-\{[(Allyloxy)carbonyl]amino\}-2,2-dimethylsuccinic acid 4-(allyl)ester 8

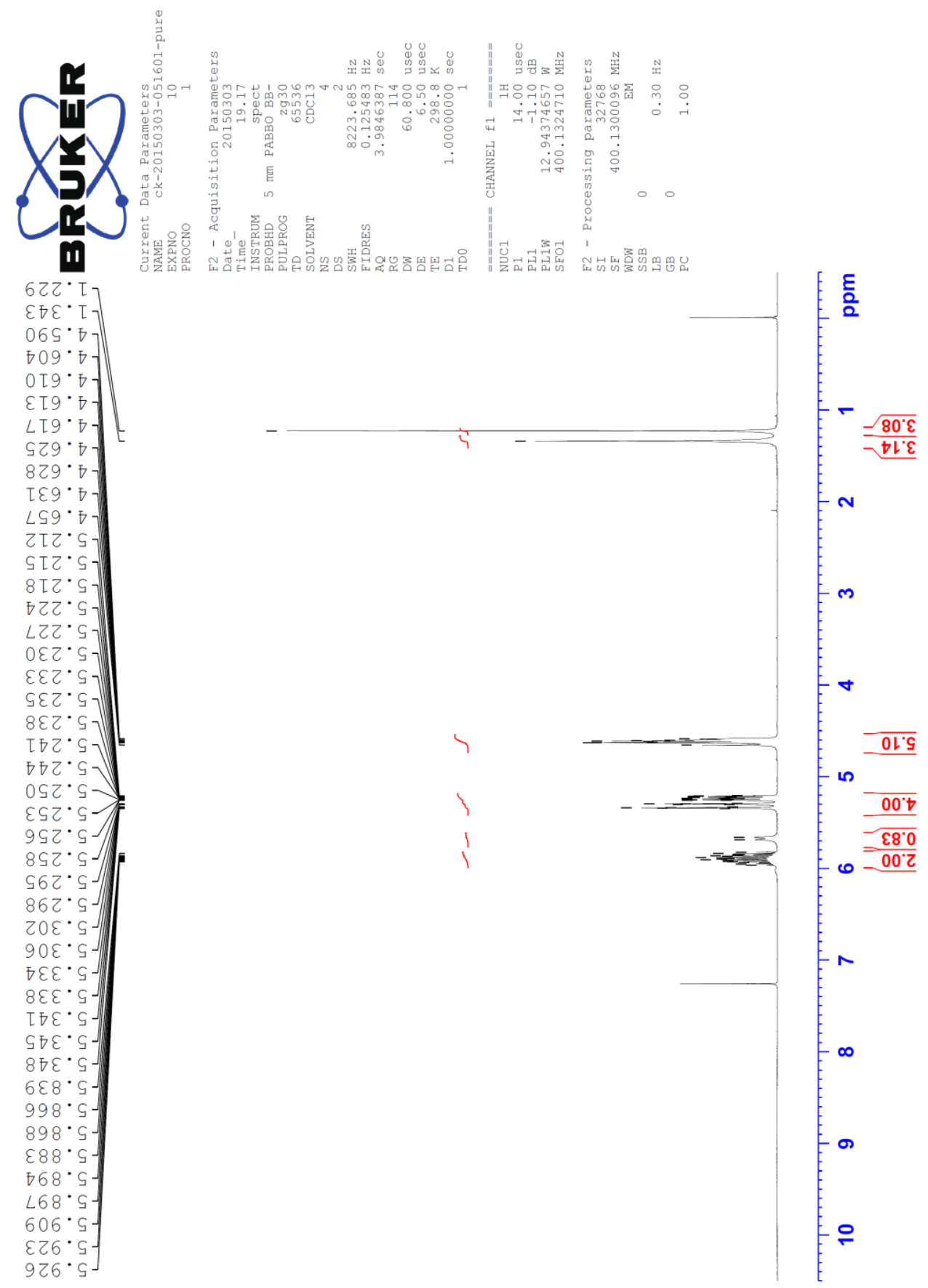




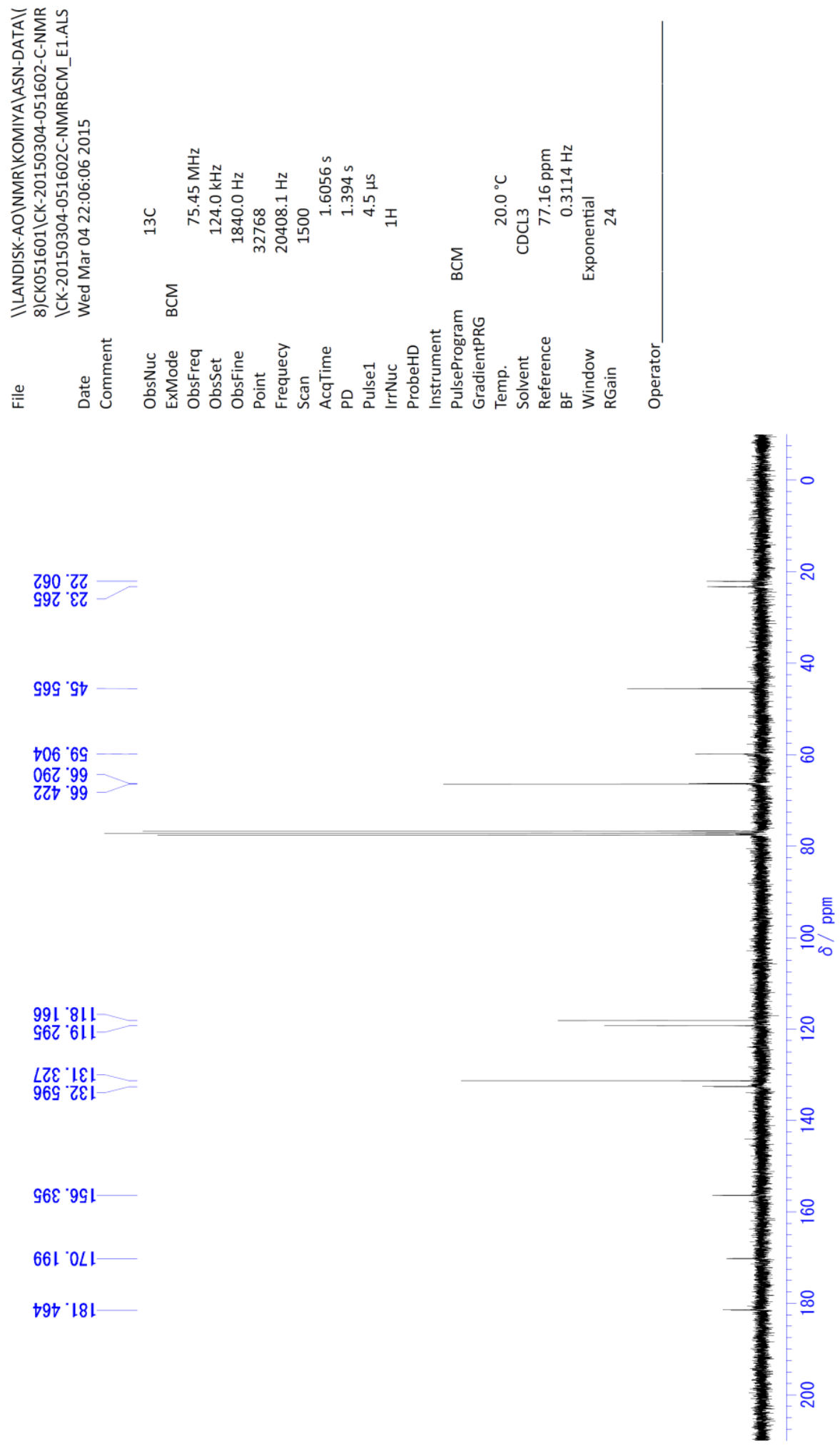




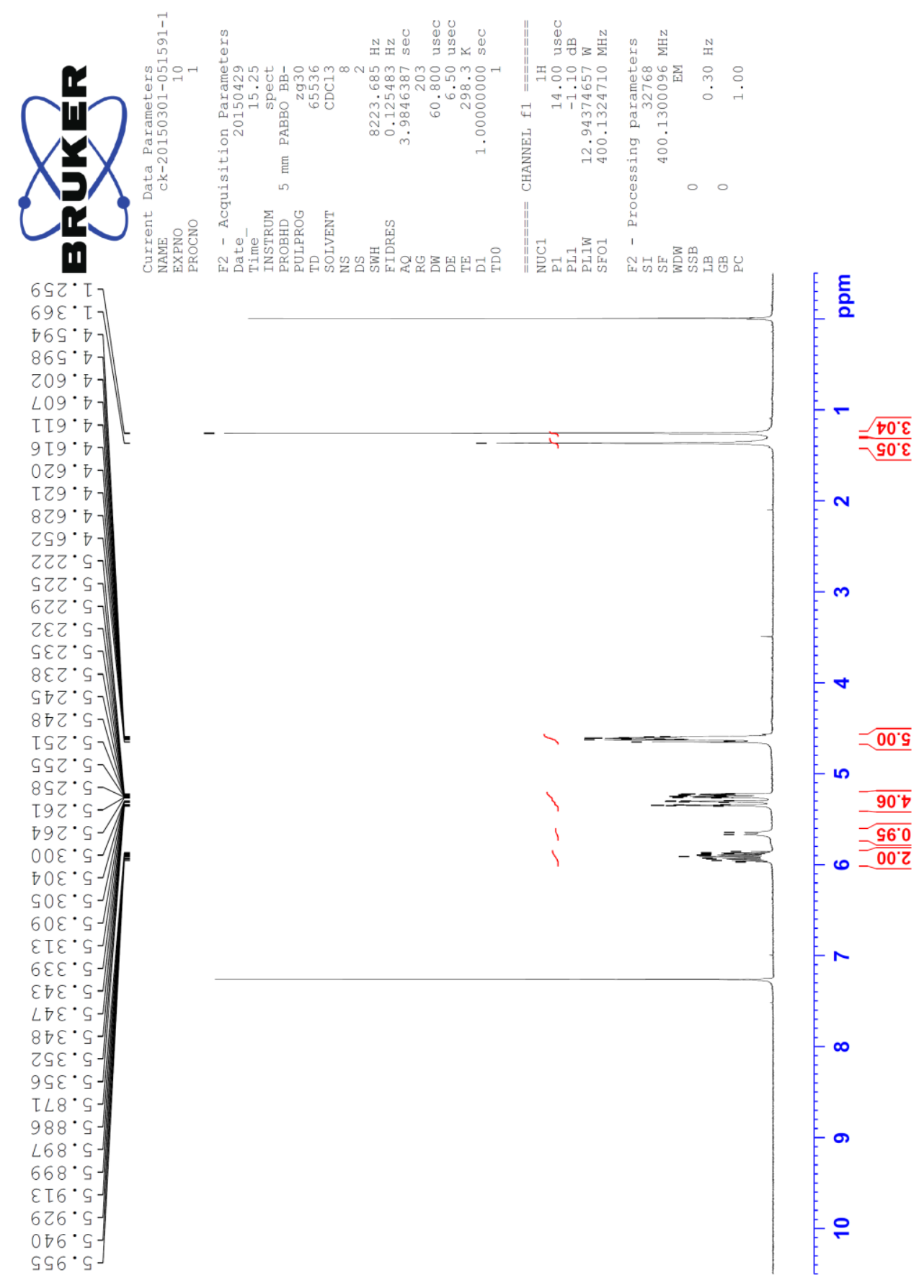




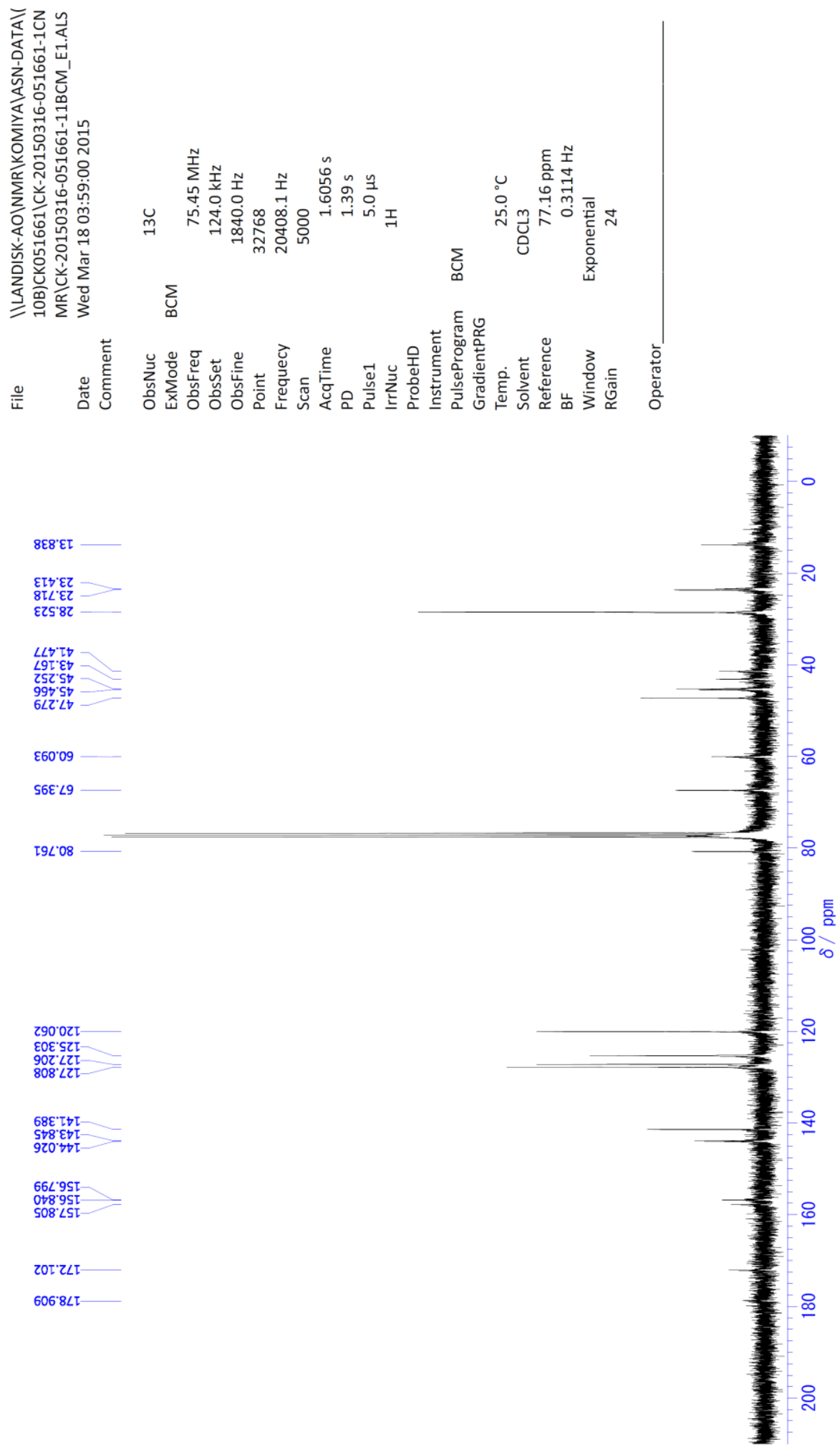


Allyl (S)-2-\{[(allyloxy)carbonyl]amino\}-4-\{[2-ethyl(2-nitrobenzyloxycarbonyl)aminoethyl]amino\}3,3-dimethyl-4-oxobutanoate 9a

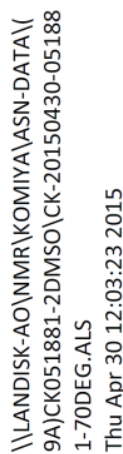

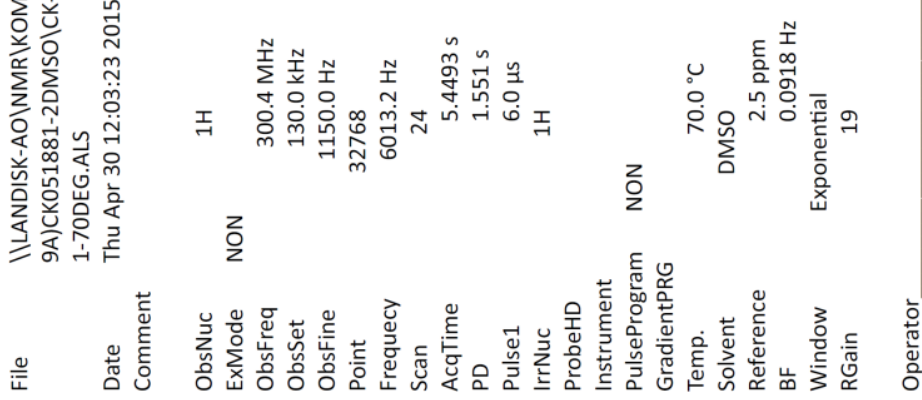

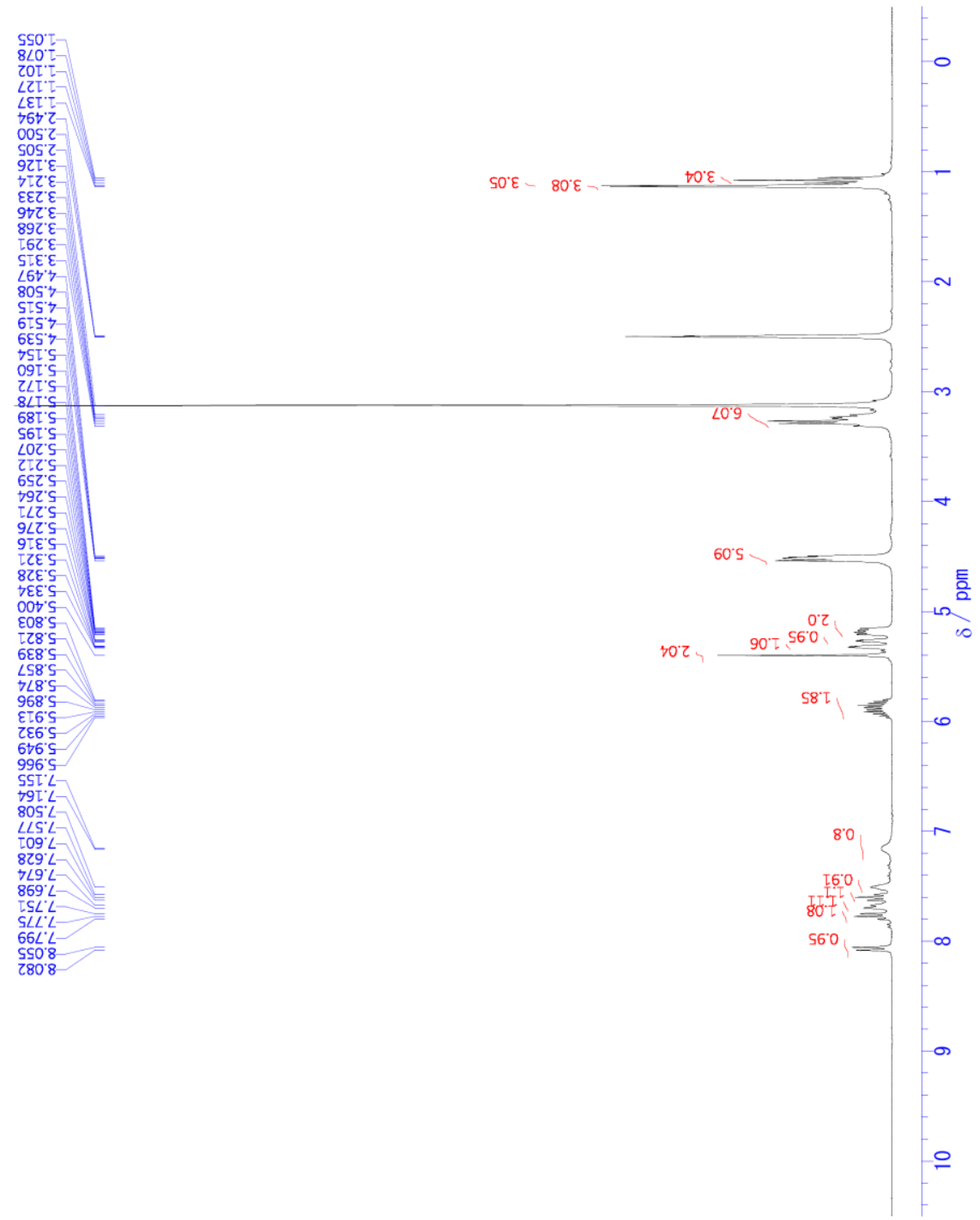



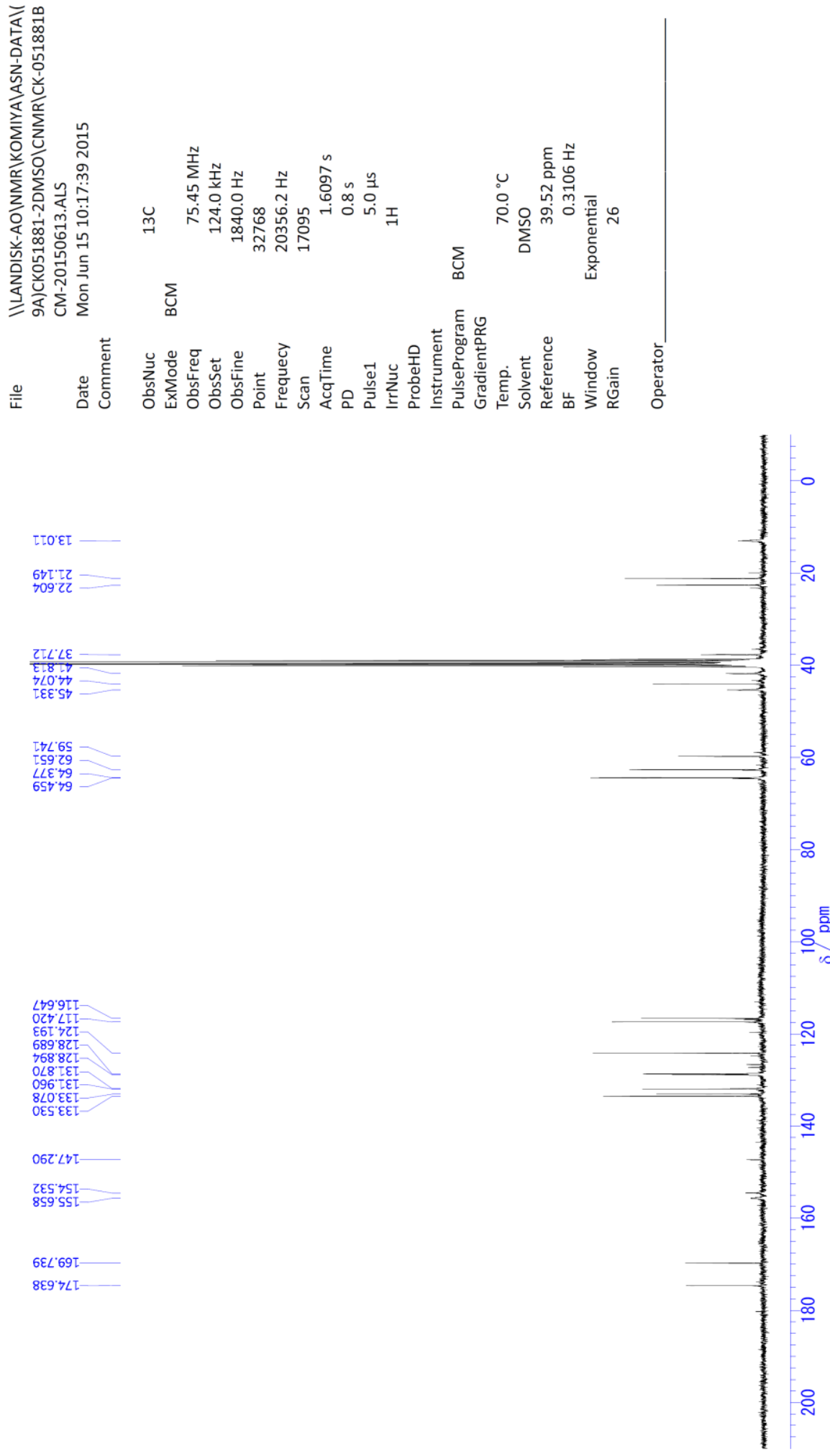
(S)-2-\{[(9H-Fluoren-9-yl)methoxycarbonyl]amino\}-4-\{[2-ethyl(2-nitrobenzyloxycarbonyl)amino ethyl]amino \}-3,3-dimethyl-4-oxobutanoic acid 10a

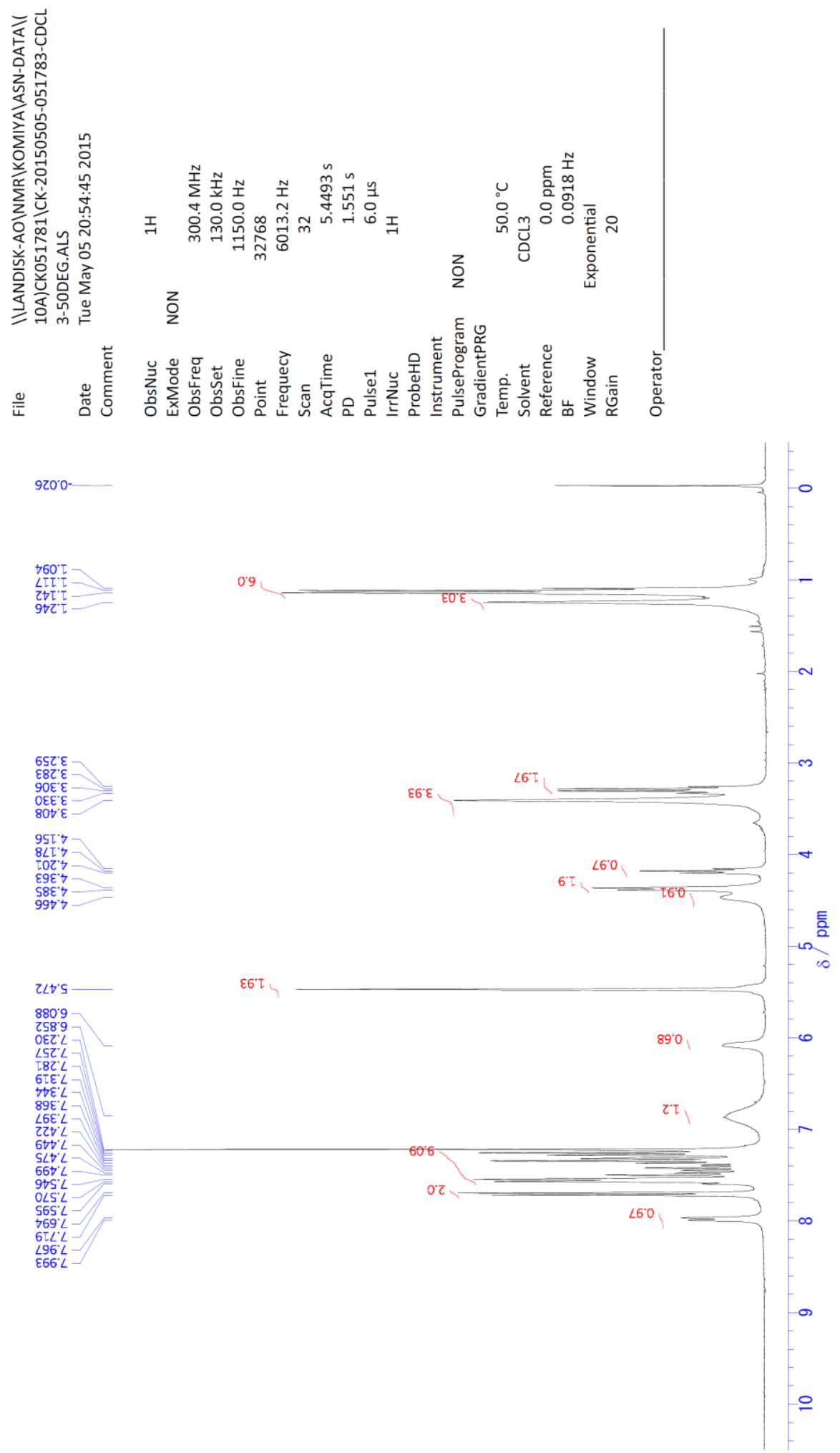




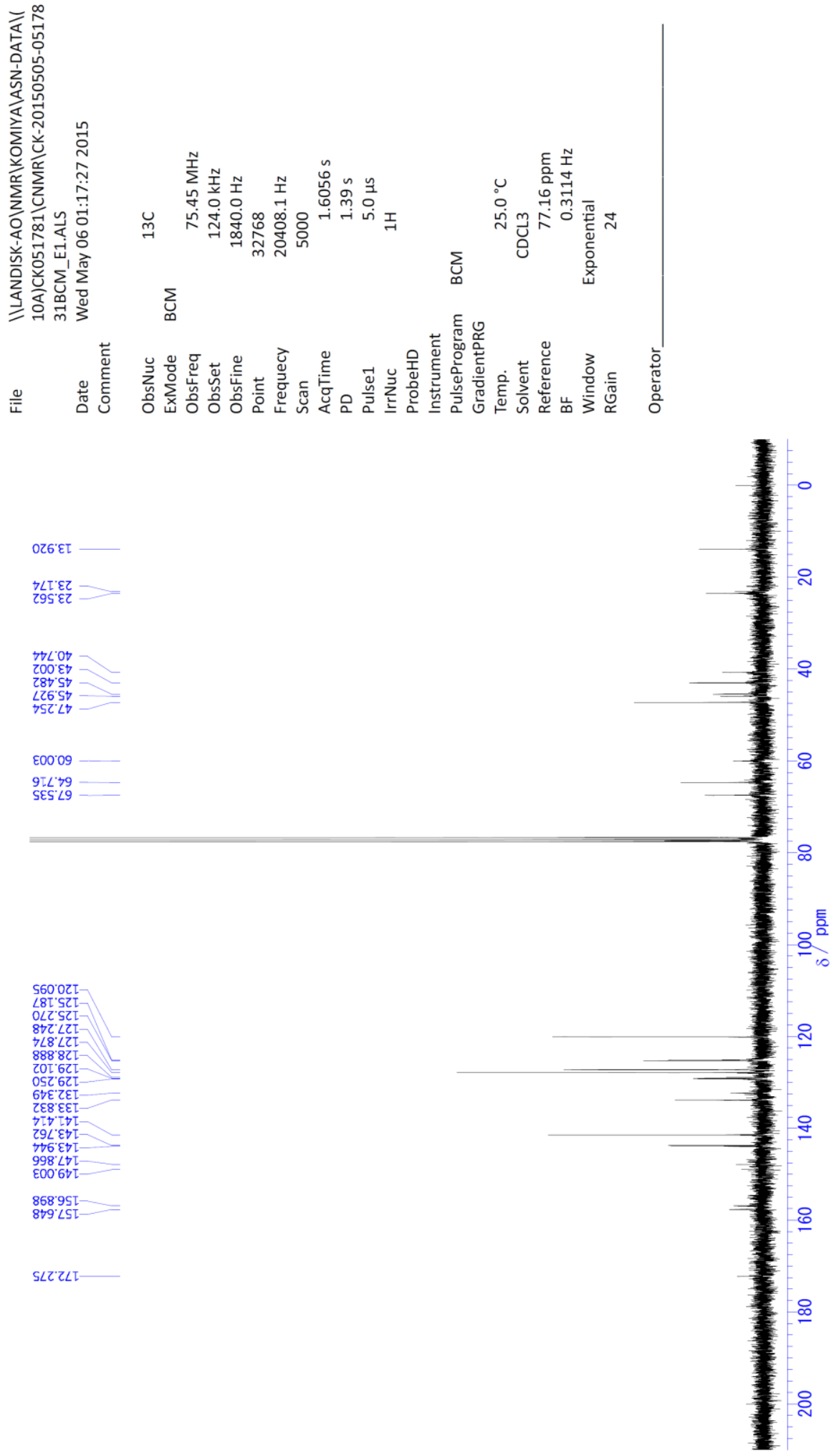


Allyl (S)-2-\{[(allyloxy)carbonyl]amino $\}-4-\{[2-($ tert-butoxycarbonyl)(ethyl)aminoethyl]amino $\}-3,3-$ dimethyl-4-oxobutanoate 9b

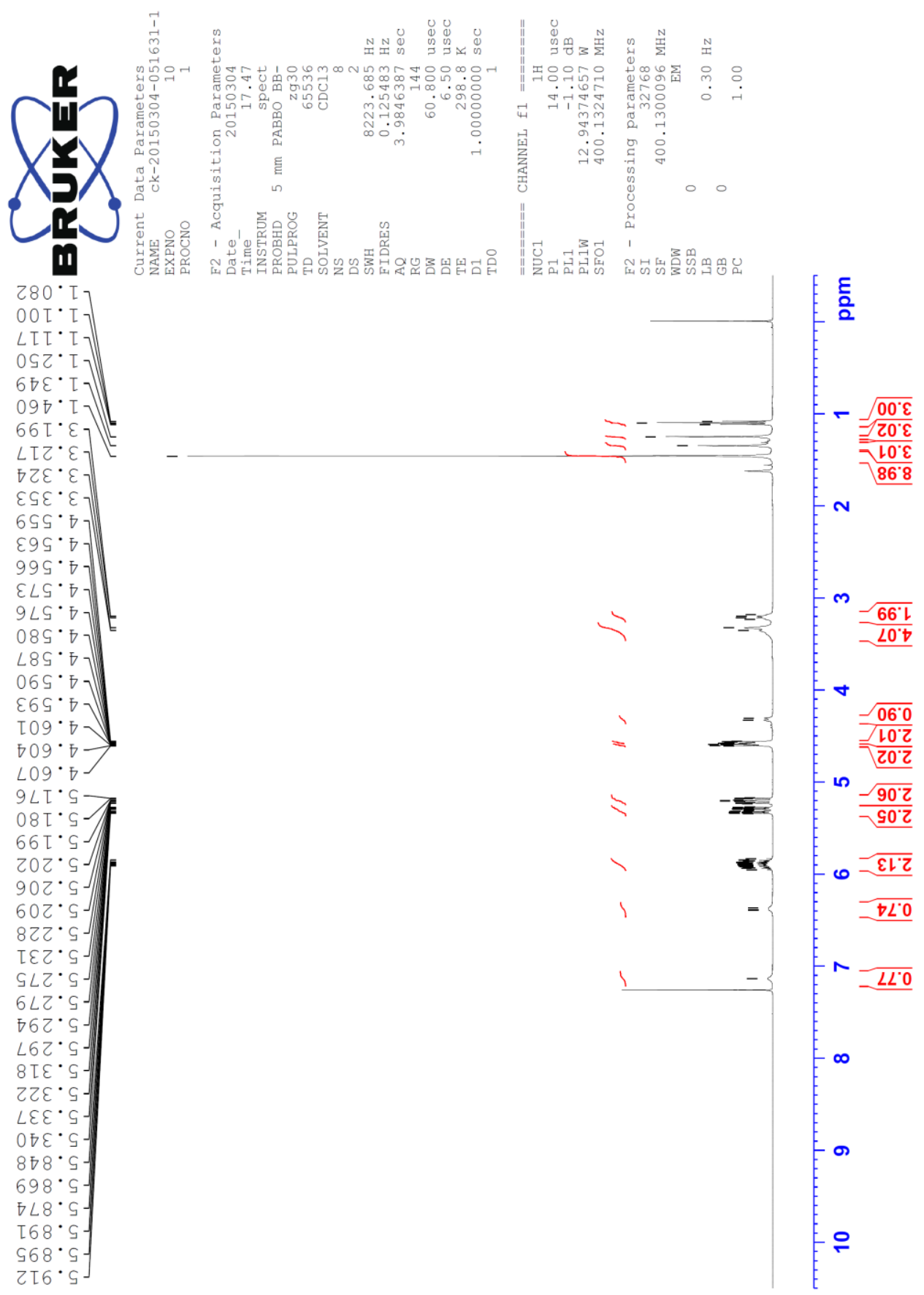




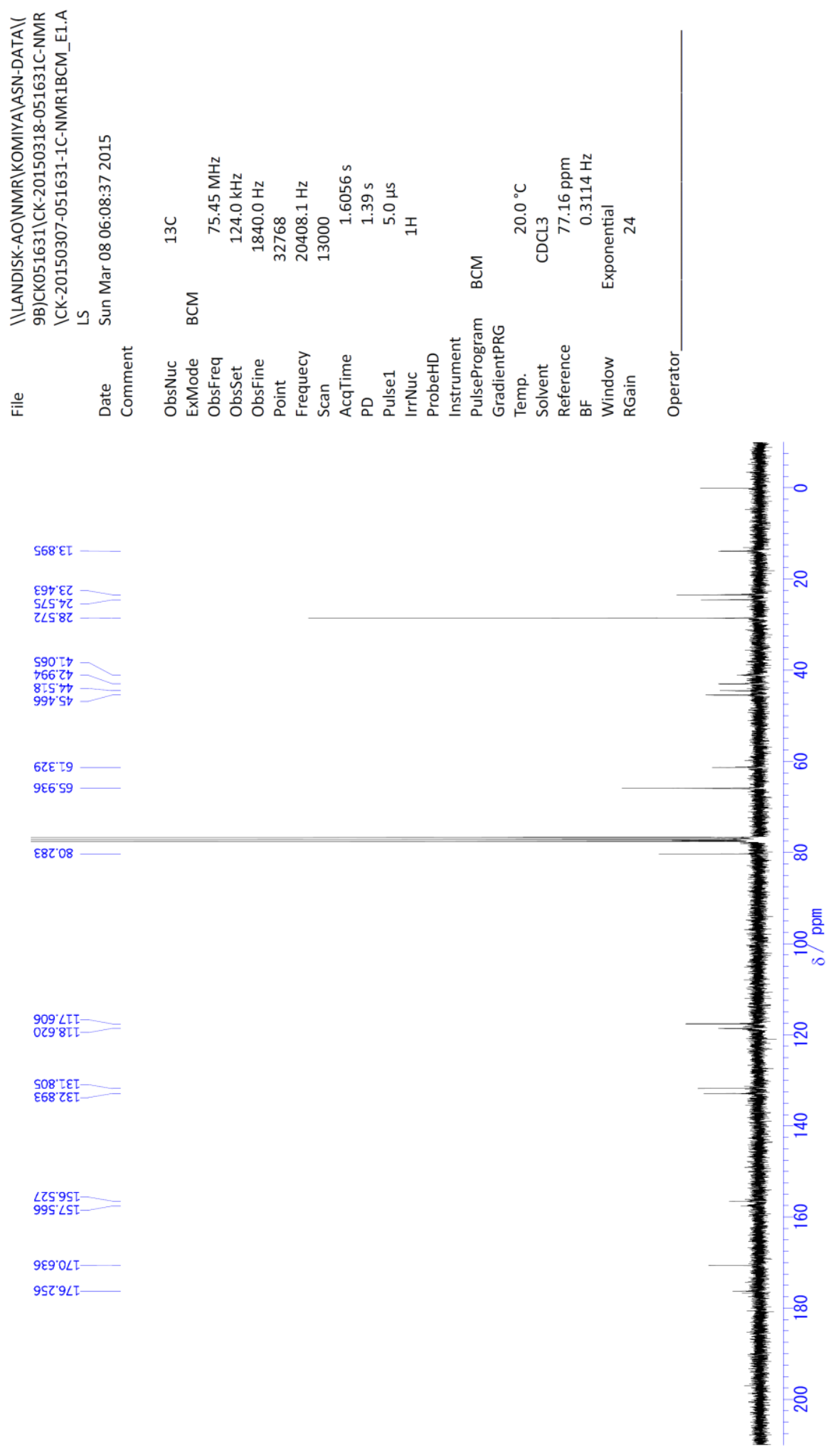


(S)-2-\{[(9H-Fluoren-9-yl)methoxycarbonyl $]$ amino $\}-4-\{[2-($ tert-butoxycarbonyl)(ethyl)aminoethyl] amino -3,3-dimethyl-4-oxobutanoic acid 10b

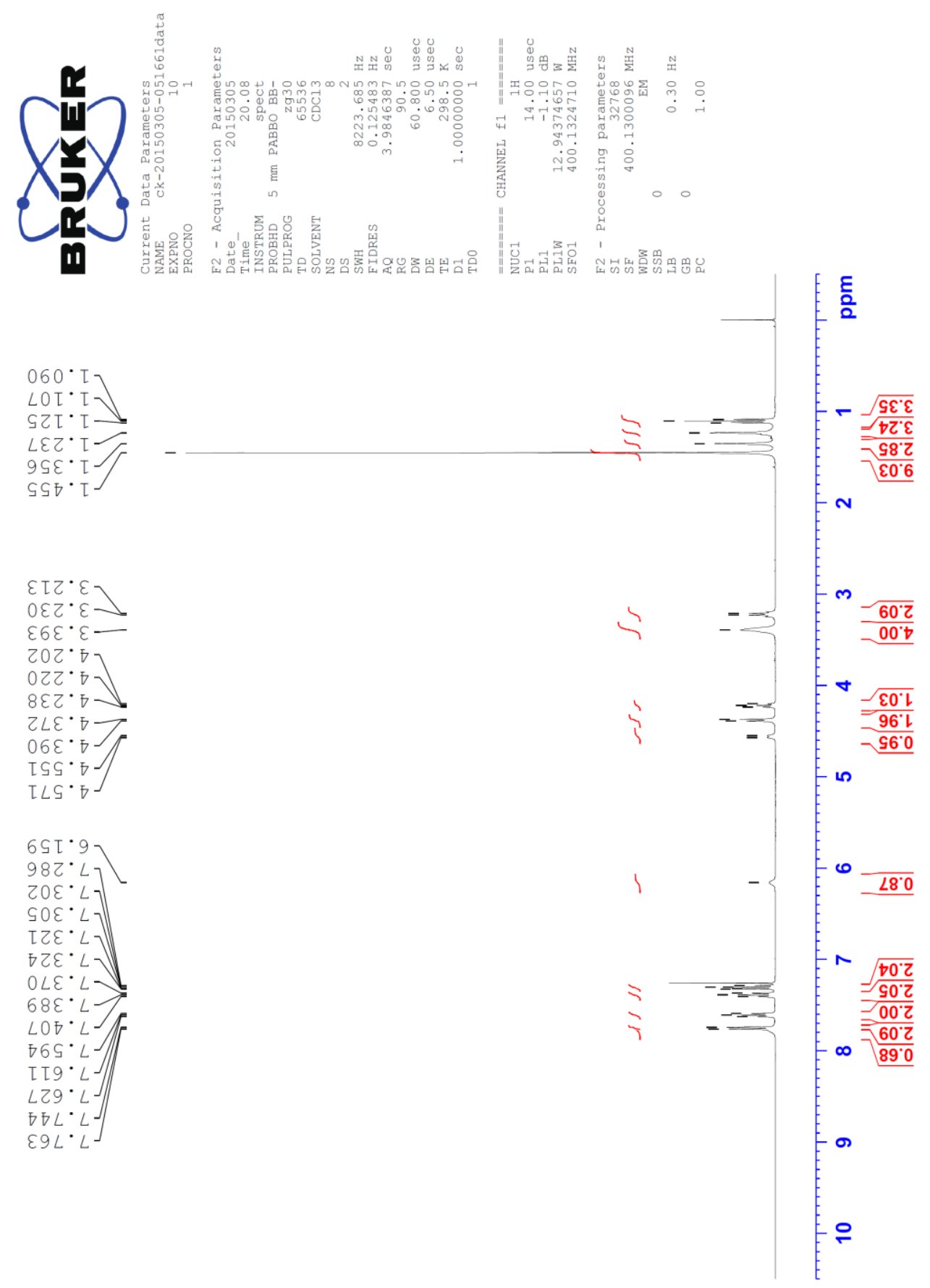




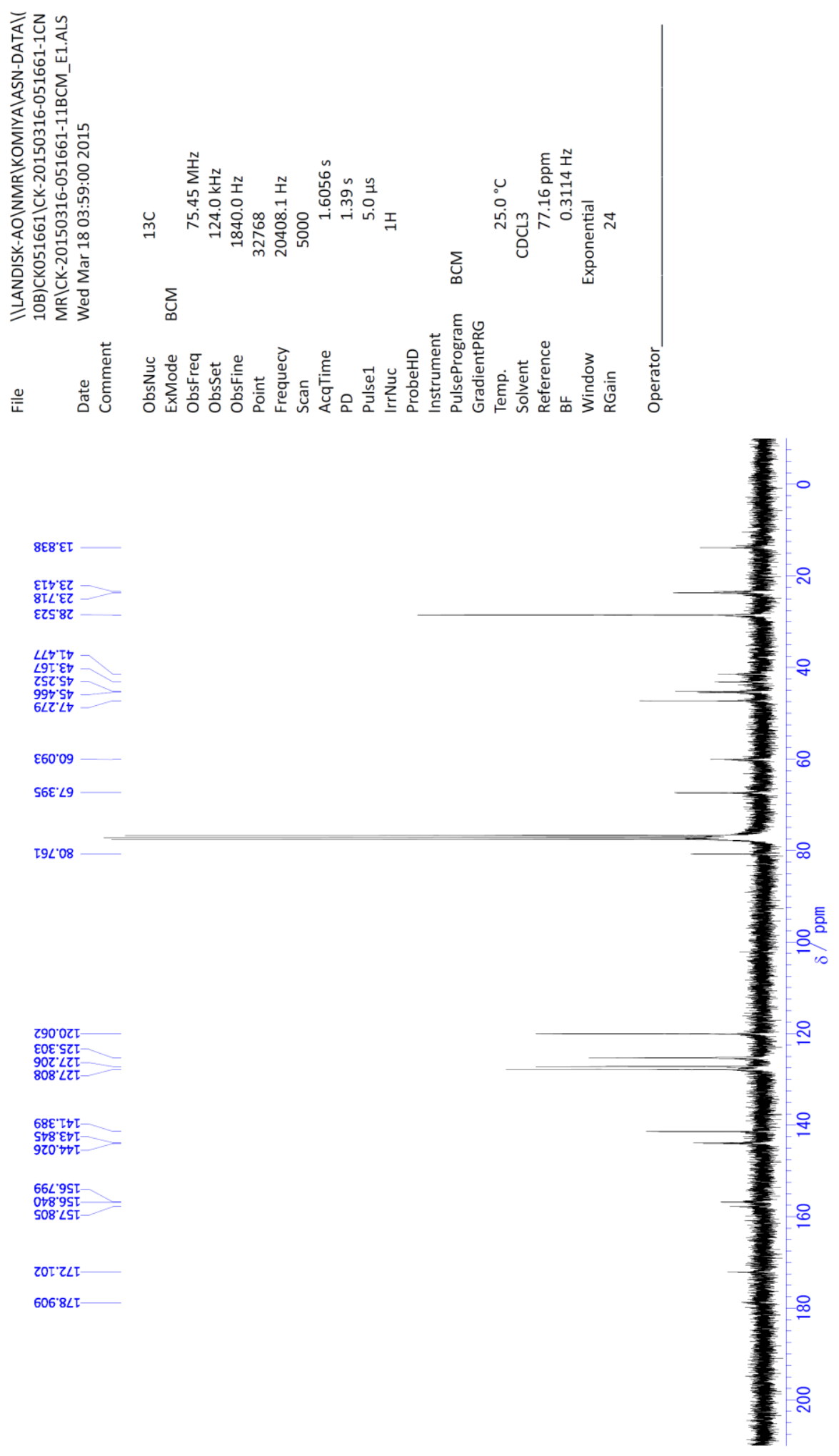


2-Nitrobenzyl \{2-[(2,4-dimethoxybenzyl)amino]ethyl\}(ethyl)carbamate 14a

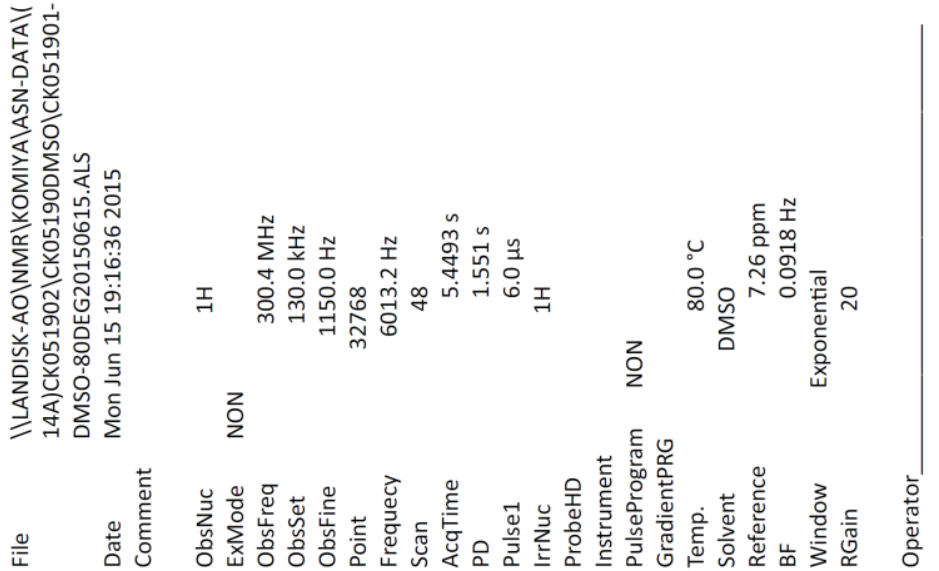

$620^{\circ} \tau-$
$\varepsilon S 0^{\circ} \tau-$
$9 \angle 0^{\circ} \tau-$
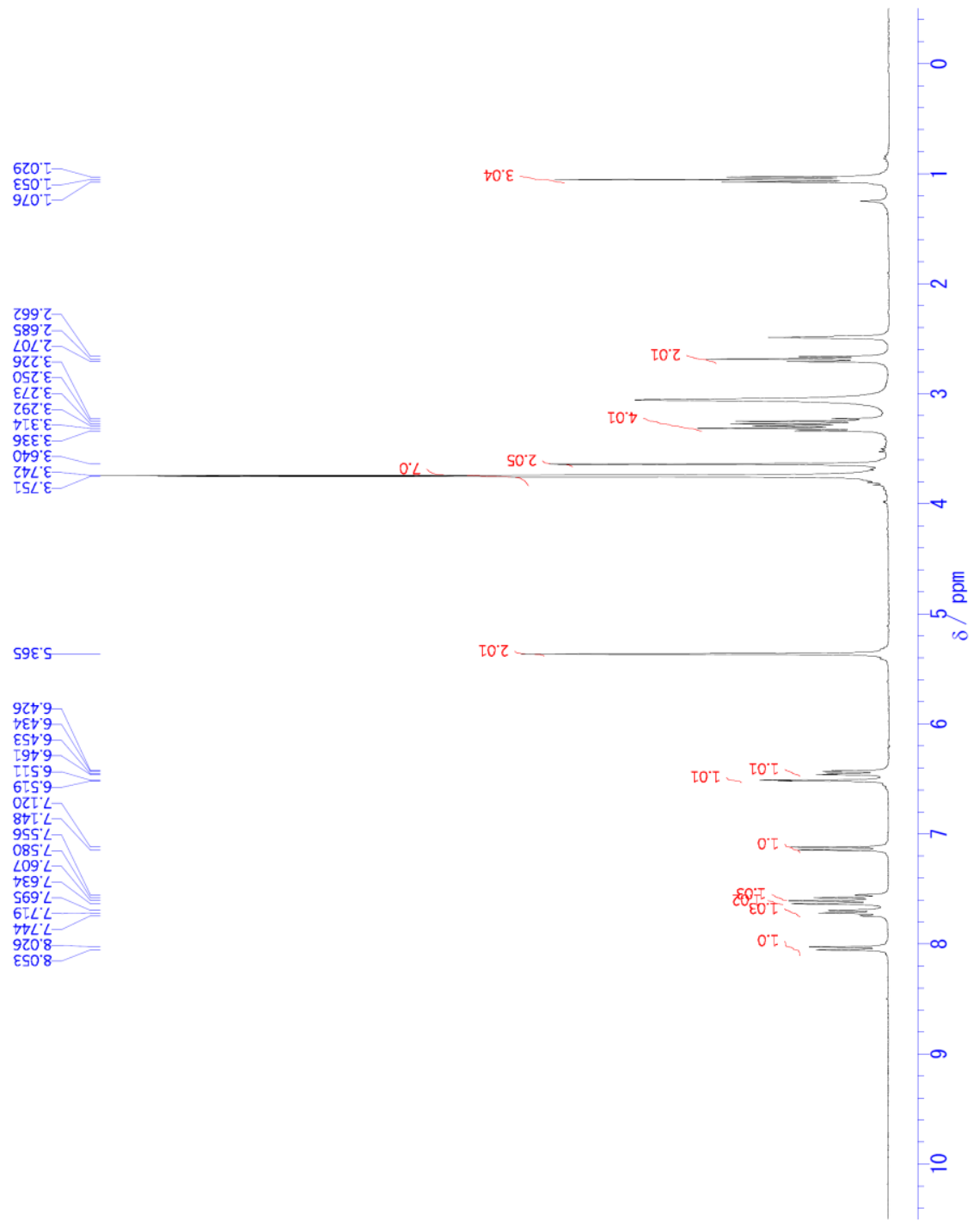


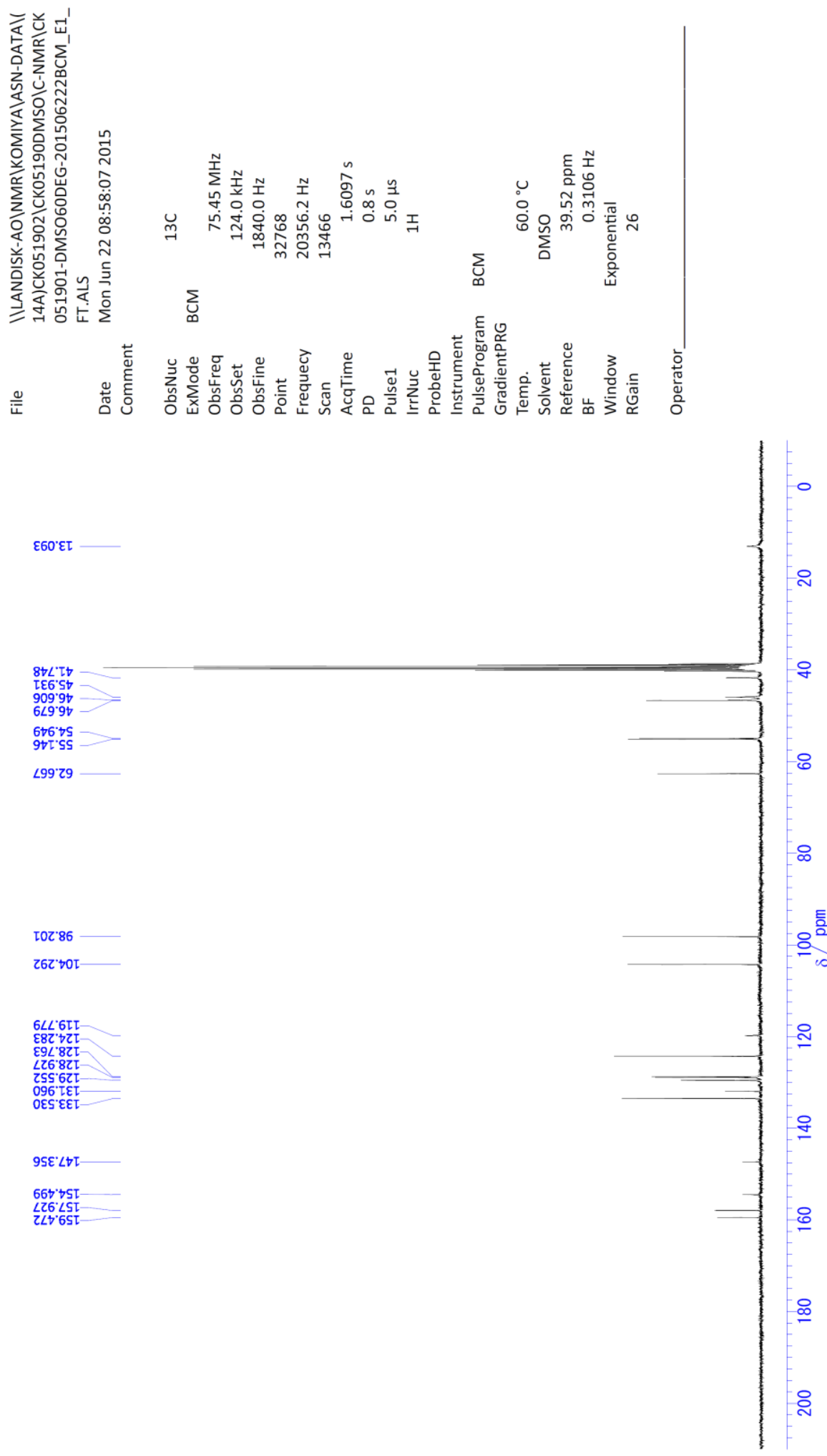


tert-Butyl $\{2-[(2,4-$ dimethoxybenzyl)amino]ethyl $\}($ ethyl)carbamate $\mathbf{1 4 b}$

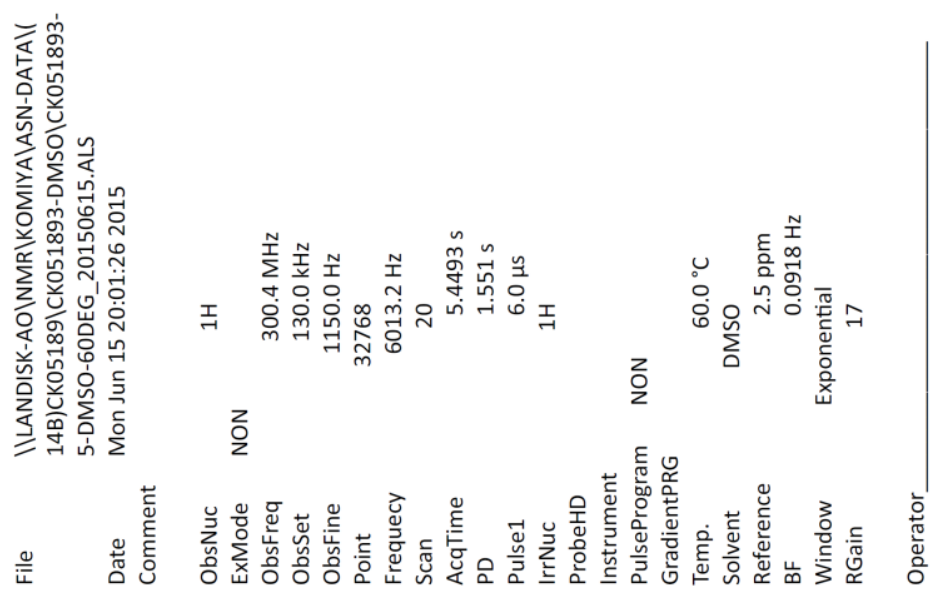

$866^{\circ} 0$
$120^{\circ} \mathrm{T}$
$\forall \rightarrow 0^{\circ} \mathrm{-}$

t99' 2
9892
$60<2$
28,2

LET:

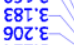

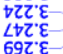

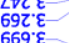

SIL'

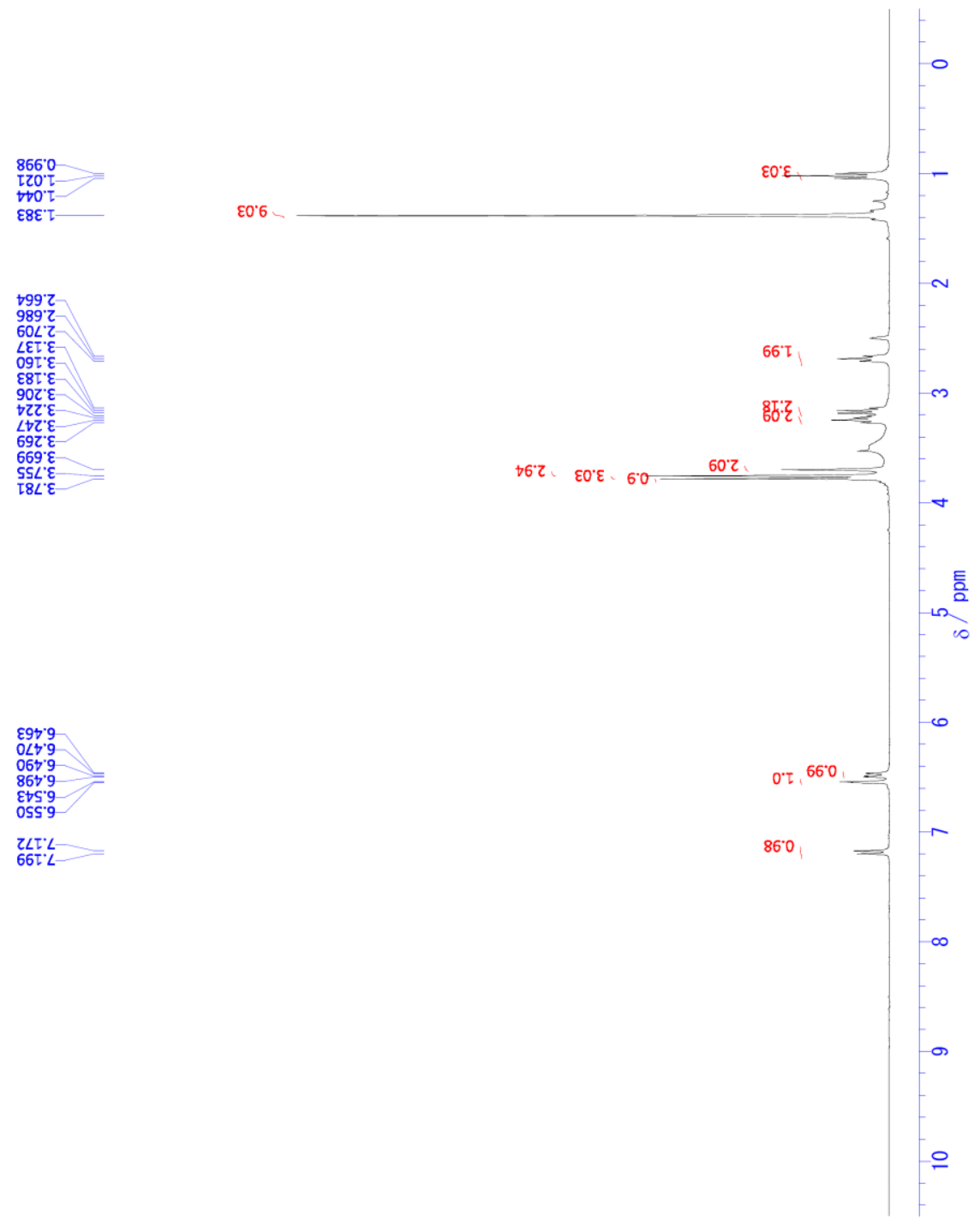




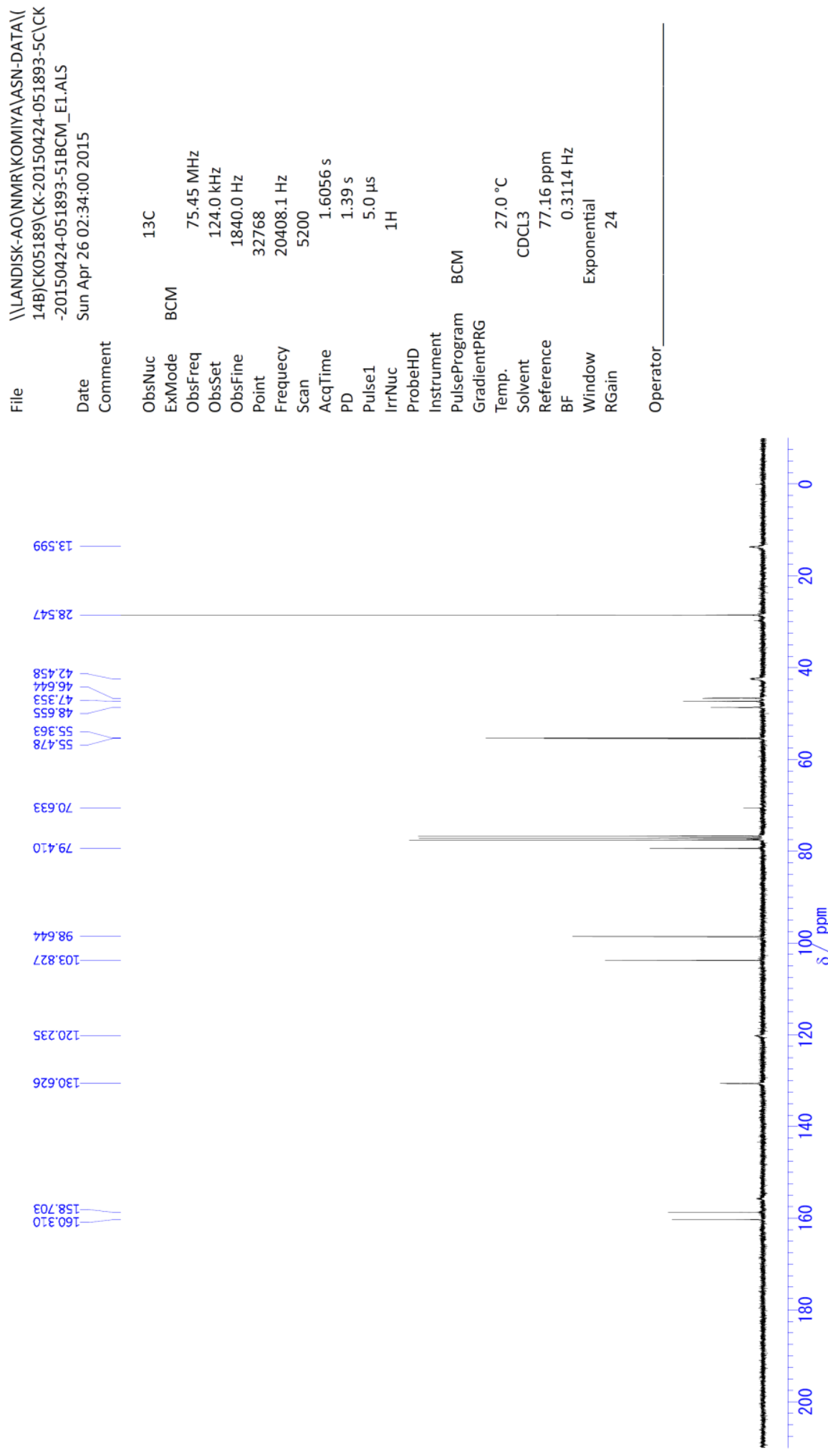


Allyl (S)-2-[(allyloxycarbonyl)amino]-4-[(2,4-dimethoxybenzyl)(2-ethyl-2-nitrobenzyloxycarbonyl aminoethyl)amino]-3,3-dimethyl-4-oxobutanoate 15

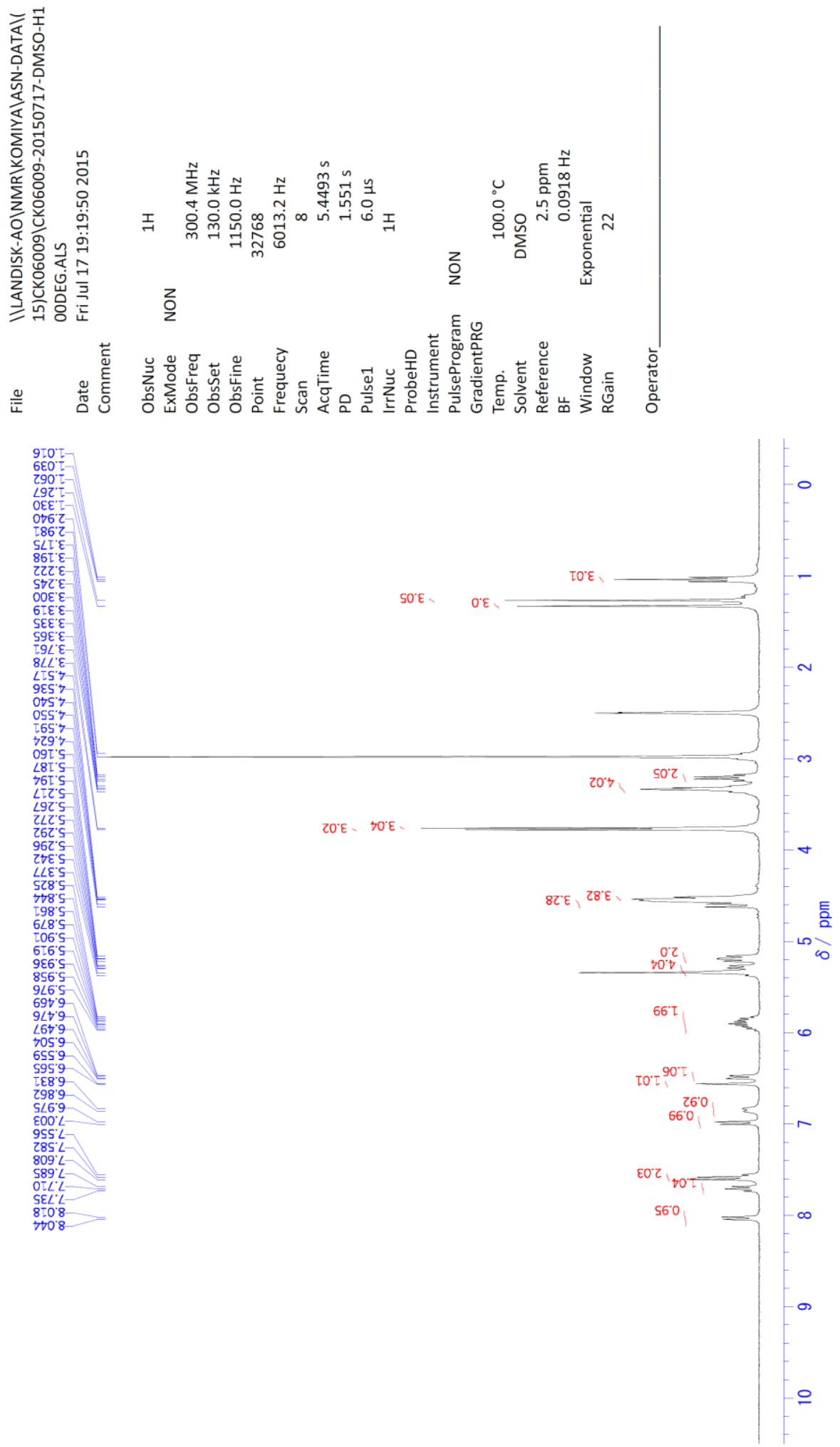




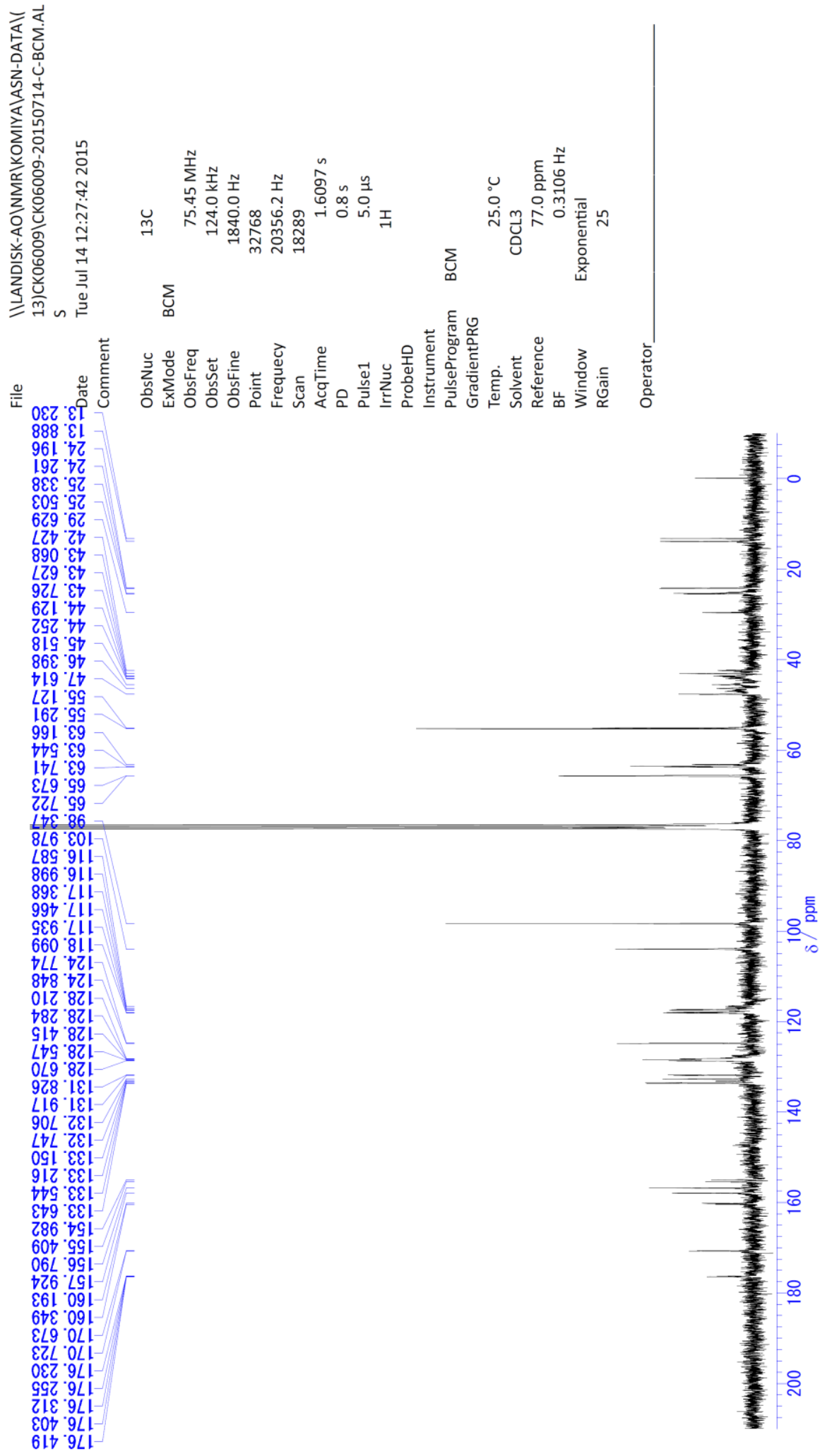


Allyl (S)-3-(allyloxycarbonyl)amino-4-\{[2,4-dimethoxybenzyl][2-(ethyl-2-nitrobenzyloxycarbonyl amino)ethyl]amino \}-2,2-dimethyl-4-oxobutanoate $\mathbf{2 5}$
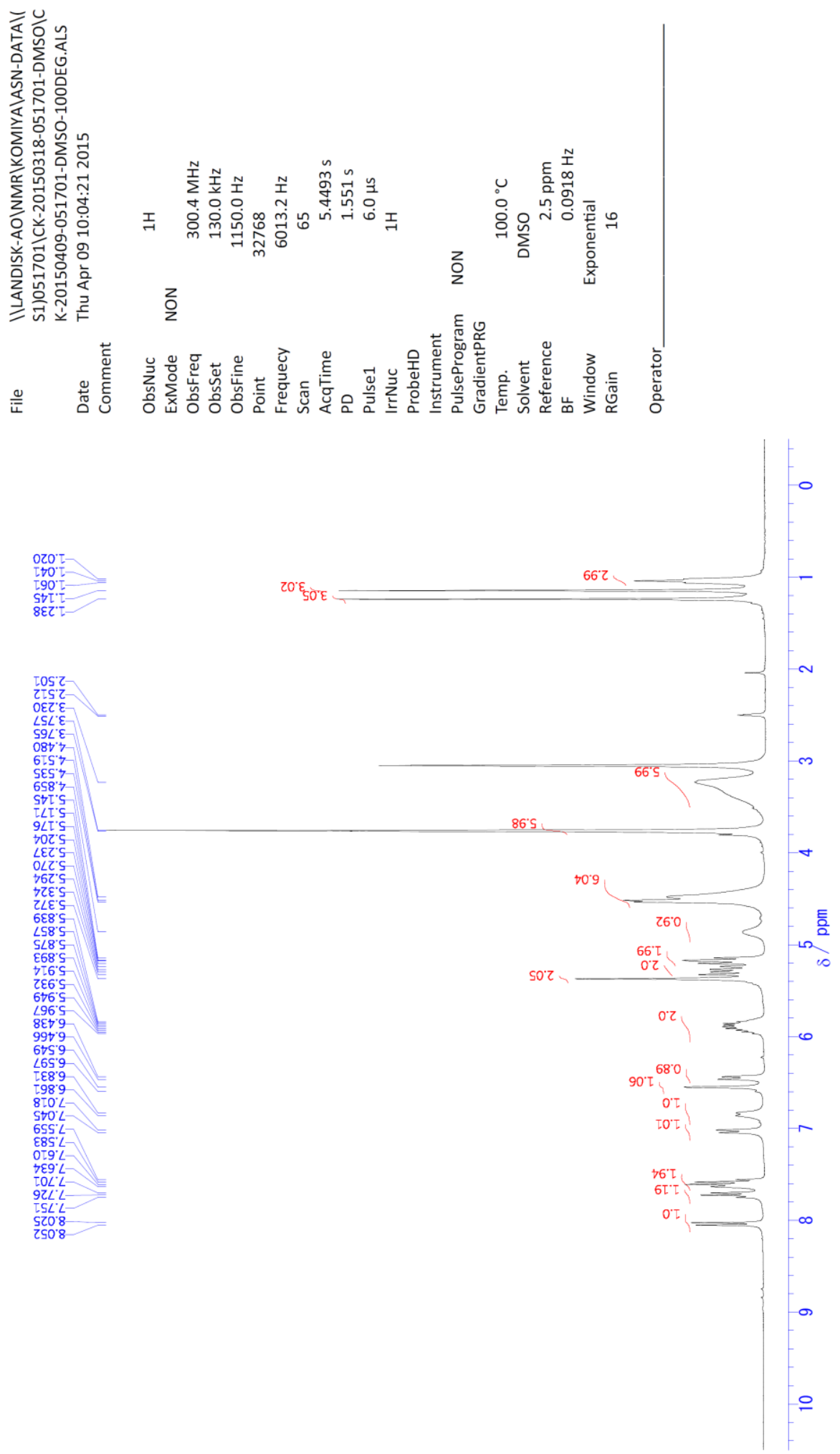


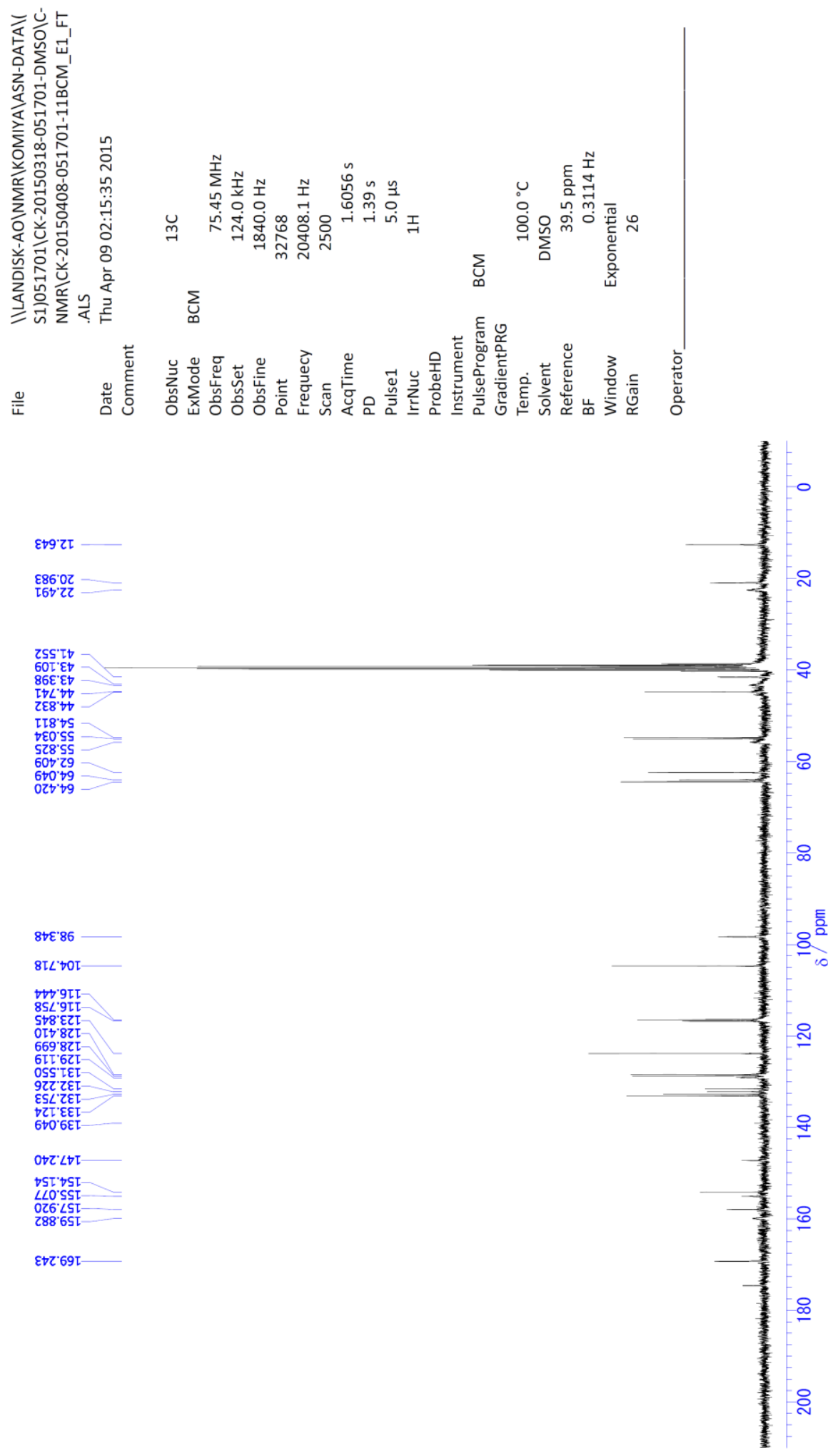


(S)-3-\{[(9H-Fluoren-9-yl)methoxycarbonyl]amino\}-4-\{[2,4-dimethoxybenzyl][2-ethyl(2-nitrobenzyl oxycarbonyl)aminoethyl]amino\}-2,2-dimethyl-4-oxobutanoic acid 16
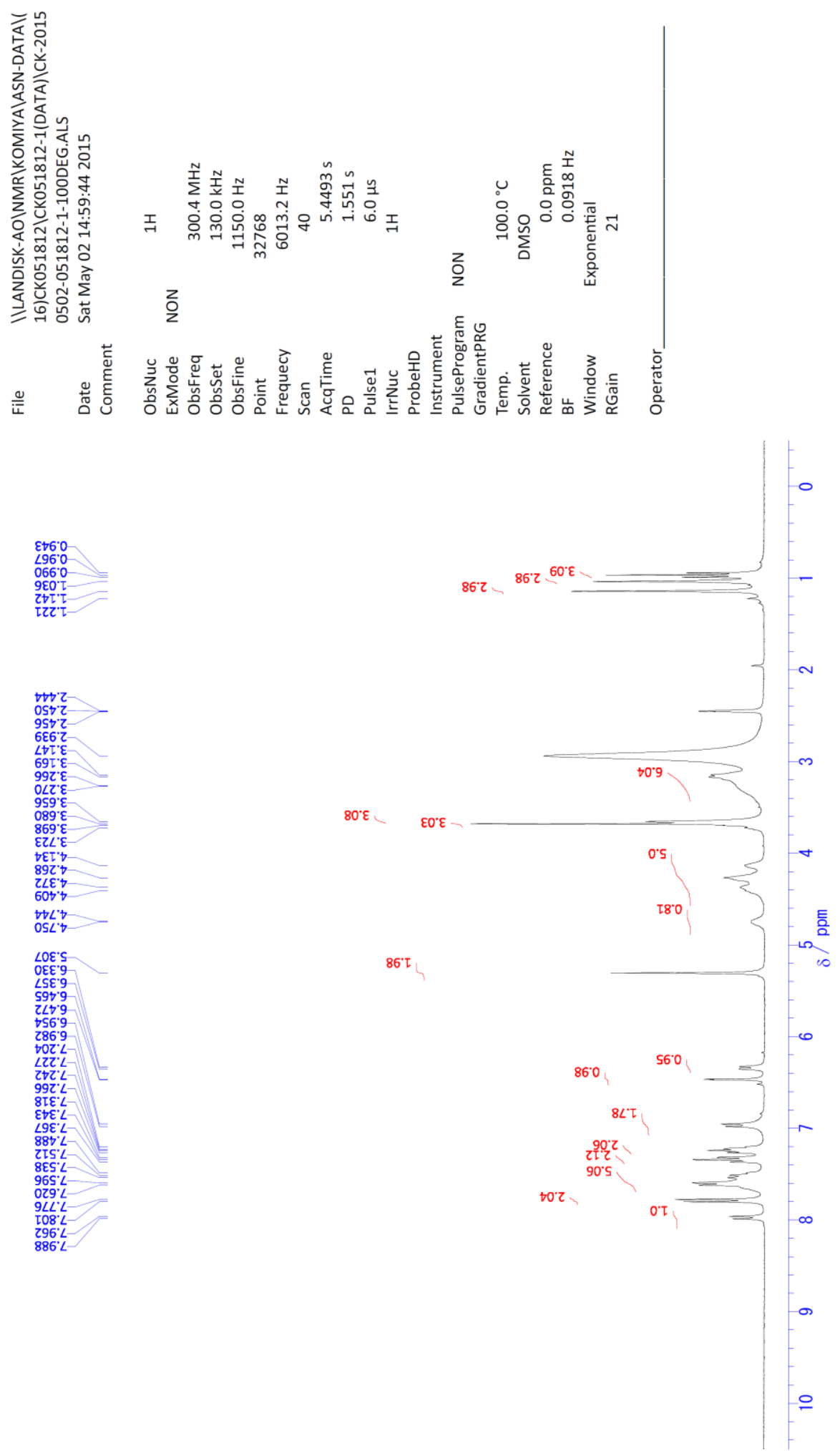

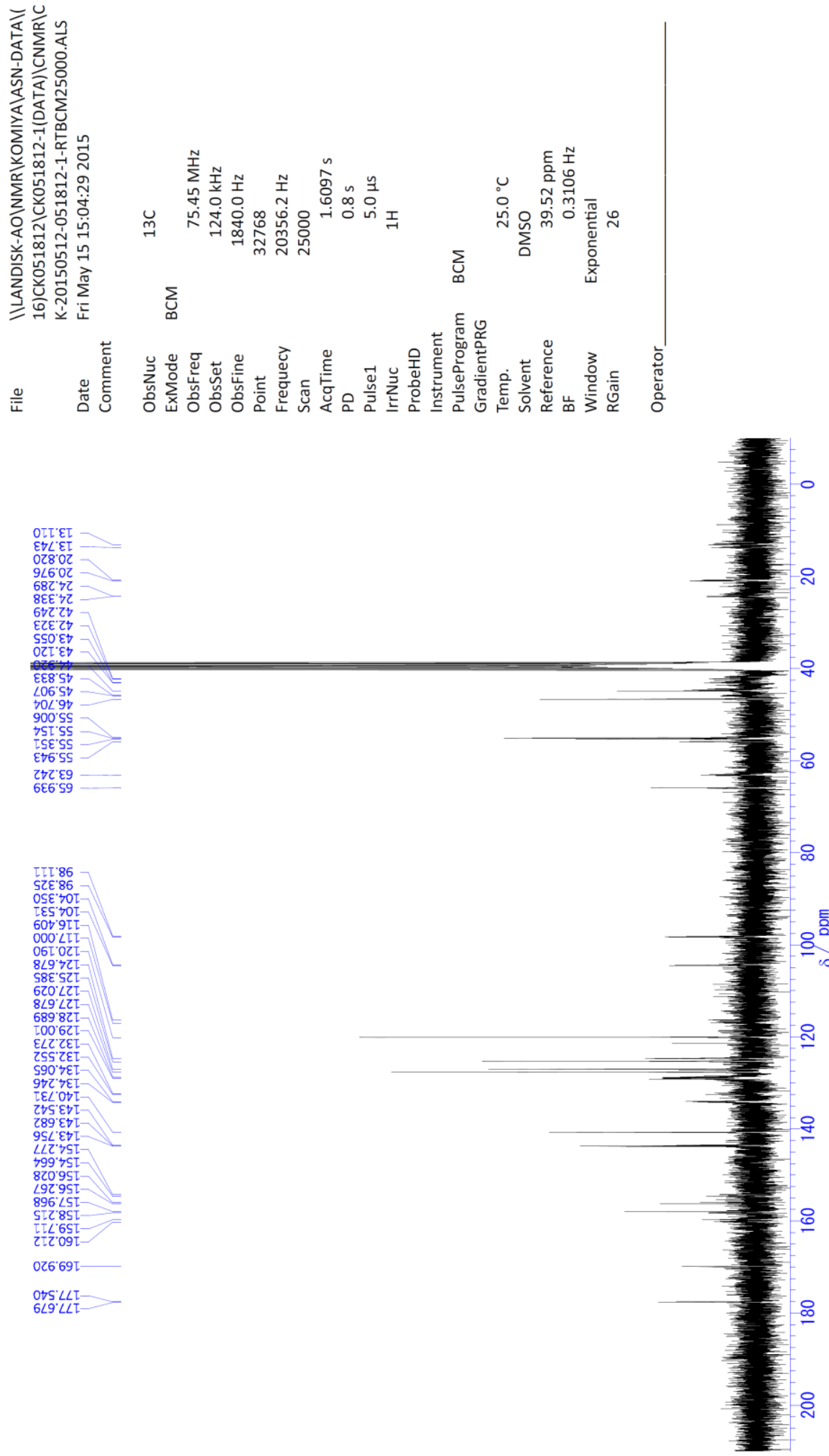
Allyl

(S)-3-[(allyloxycarbonyl)amino]-4-\{[2-ethyl(tert-butoxycarbonyl)aminoethyl][2,4dimethoxybenzyl]amino\}-2,2-dimethyl-4-oxobutanoate 26
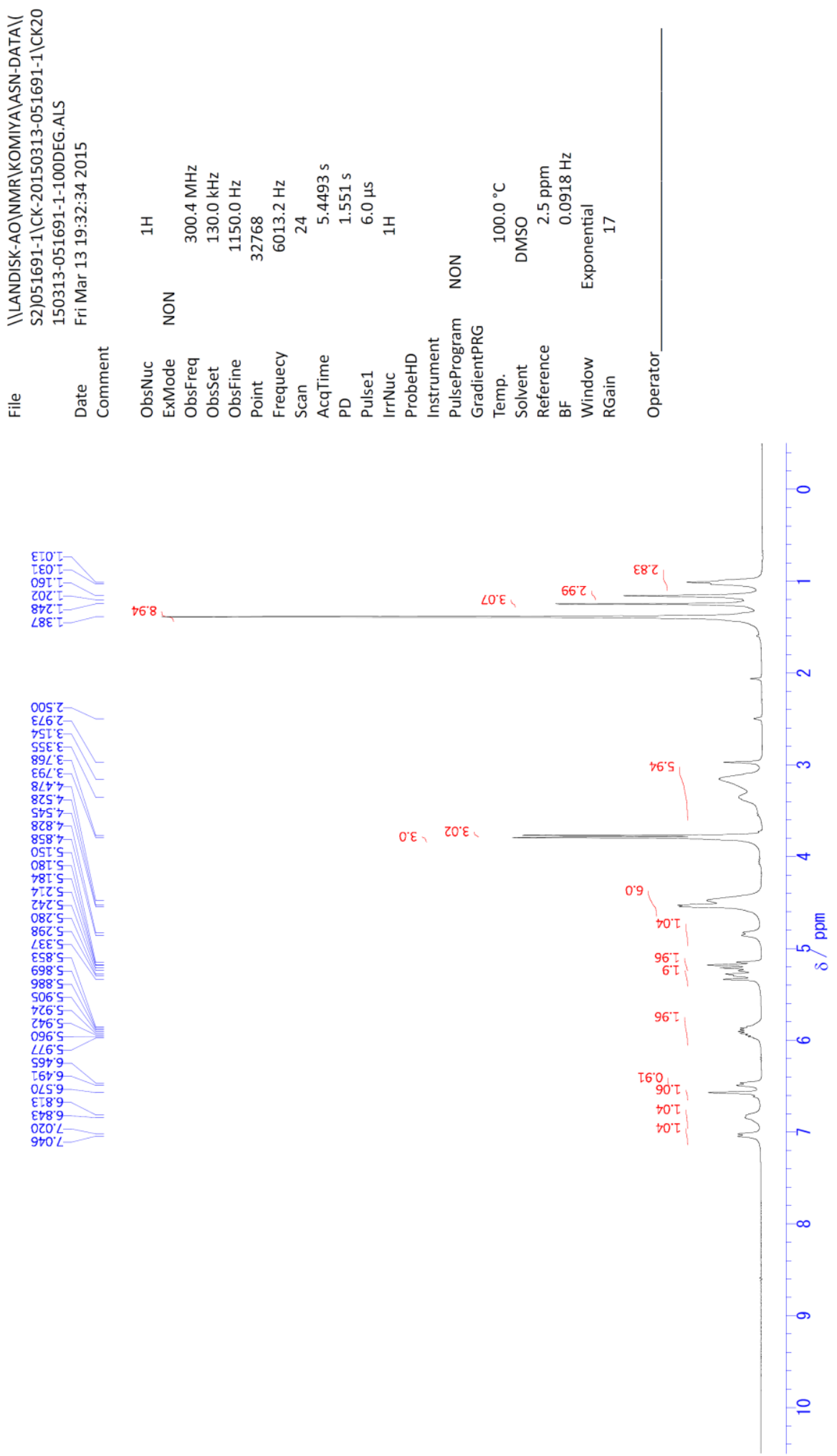


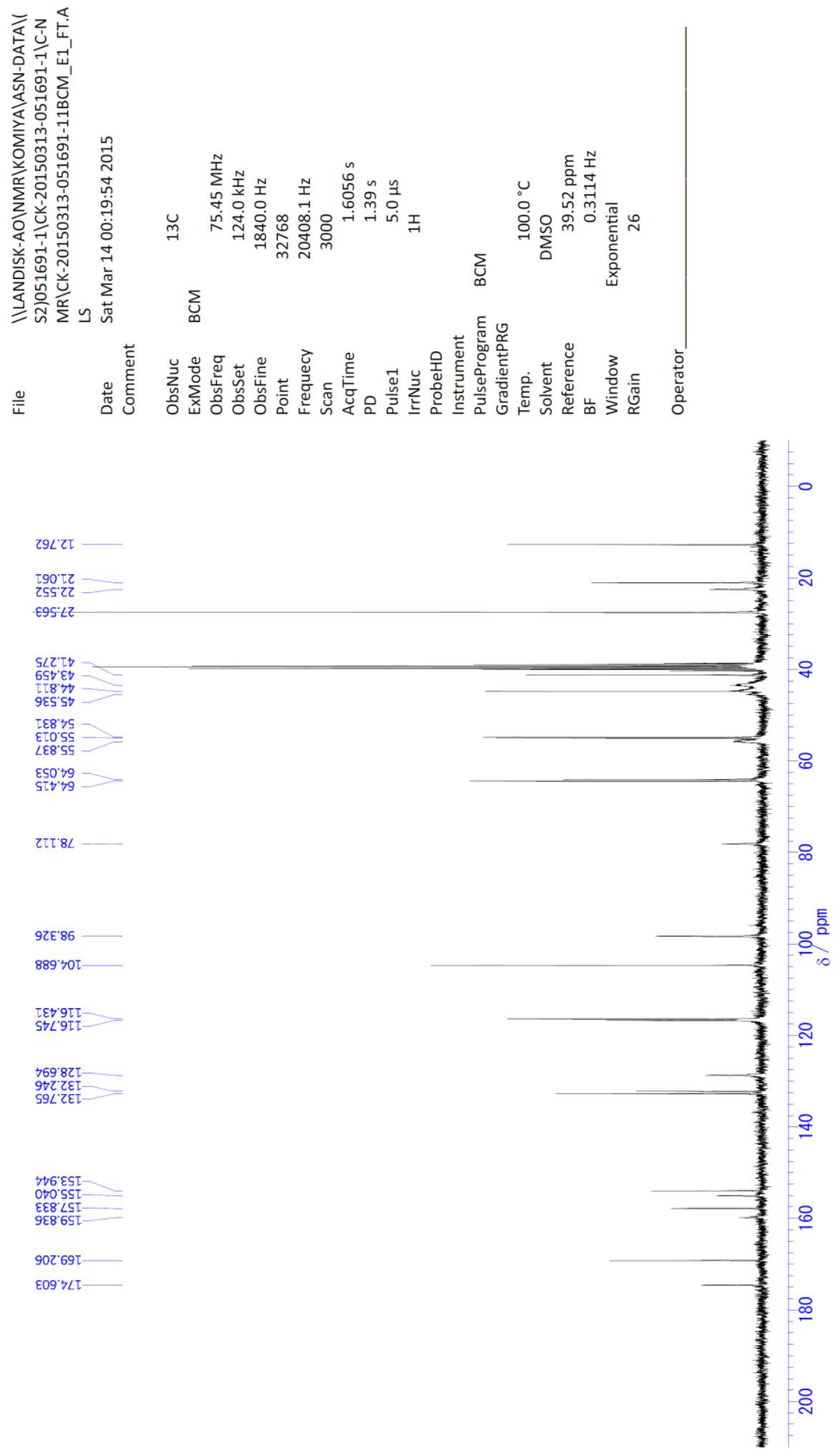


(S)-3-\{[(9H-Fluoren-9-yl)methoxycarbonyl]amino $\}-4-\{[2$-ethyl(tert-butoxycarbonyl)aminoethyl] [2,4-dimethoxybenzyl]amino -2,2-dimethyl-4-oxobutanoic acid 27
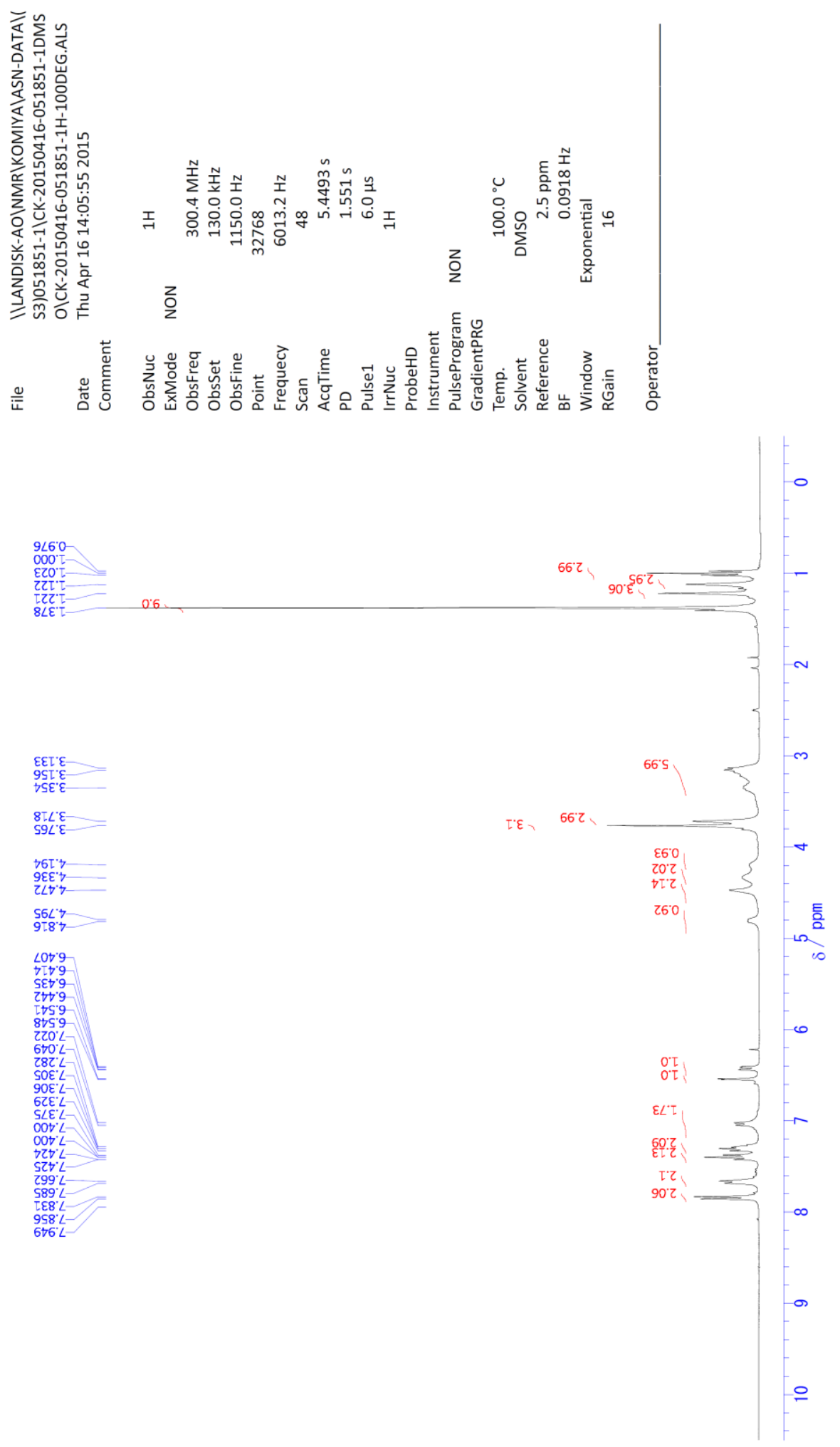

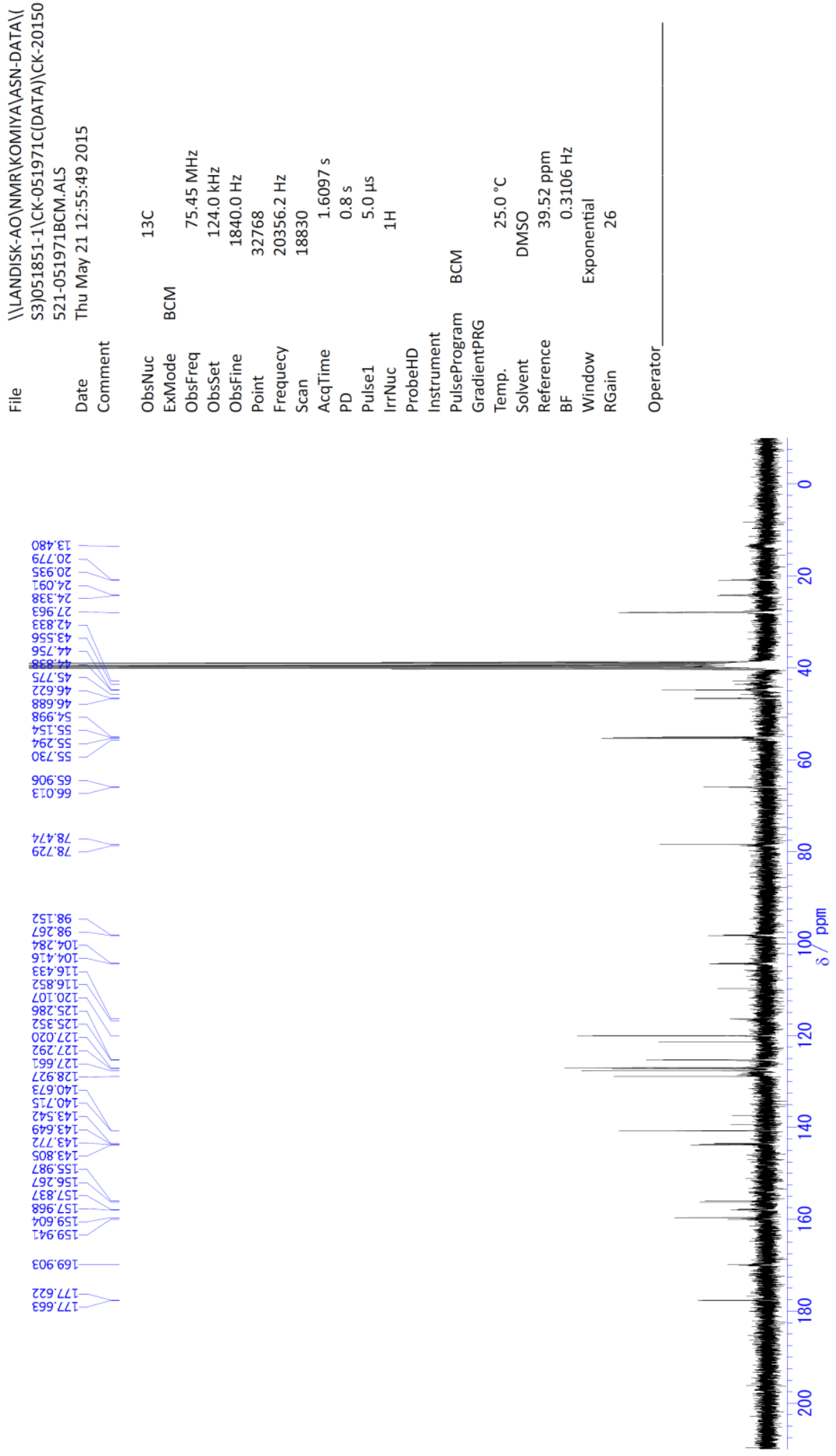
Allyl $\mathrm{N}^{2}-\left\{\left[(9 \mathrm{H}-\right.\right.$ Fluoren-9-yl)methoxy]carbonyl $\}-\mathrm{N}^{4}-\{2-[($ tert-butoxycarbonyl)(ethyl)amino $]$ ethyl $\}-$ $\mathrm{N}^{4}$-(2,4-dimethoxybenzyl)- $L$-asparaginate $\mathbf{2 8}$

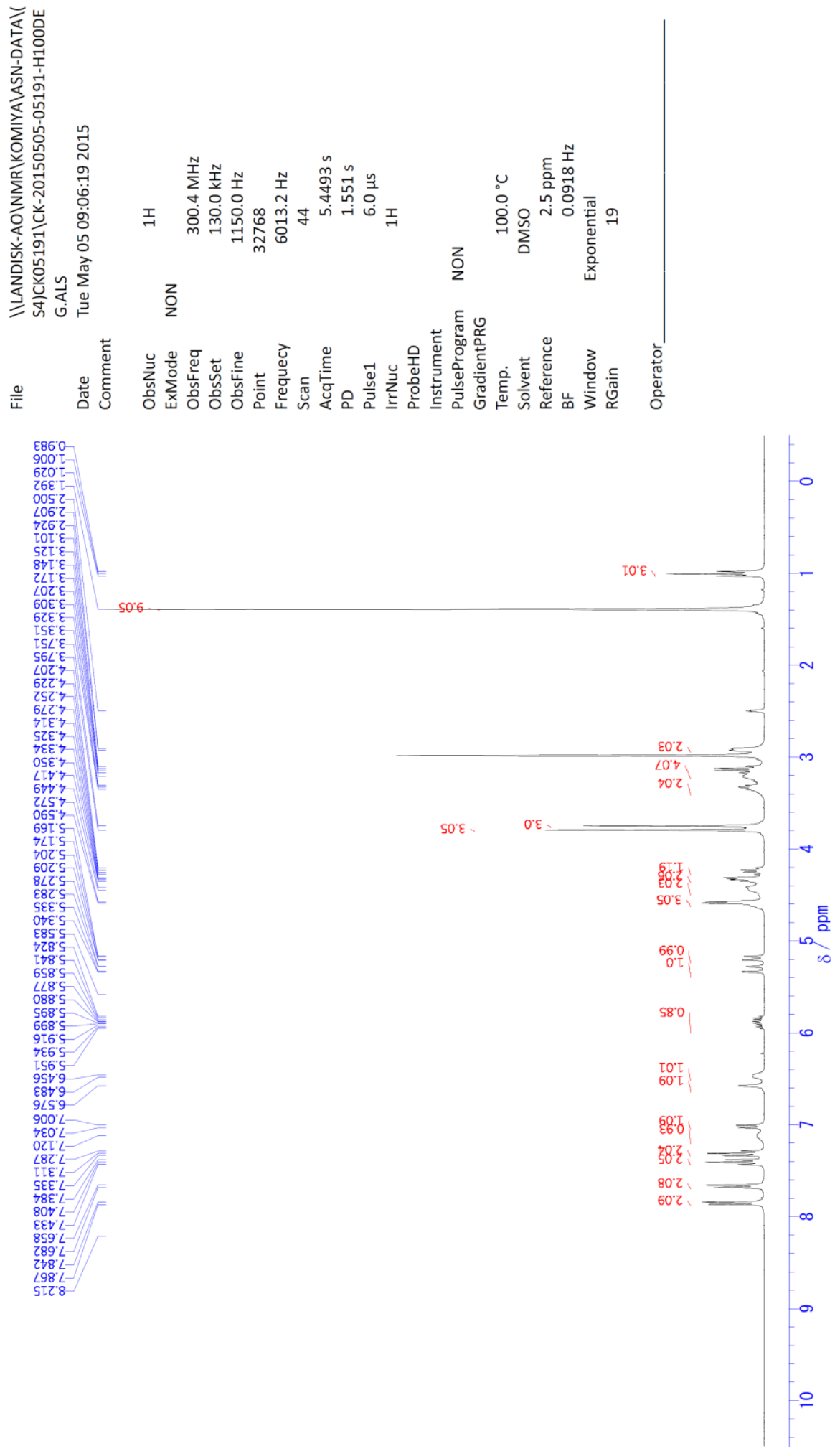



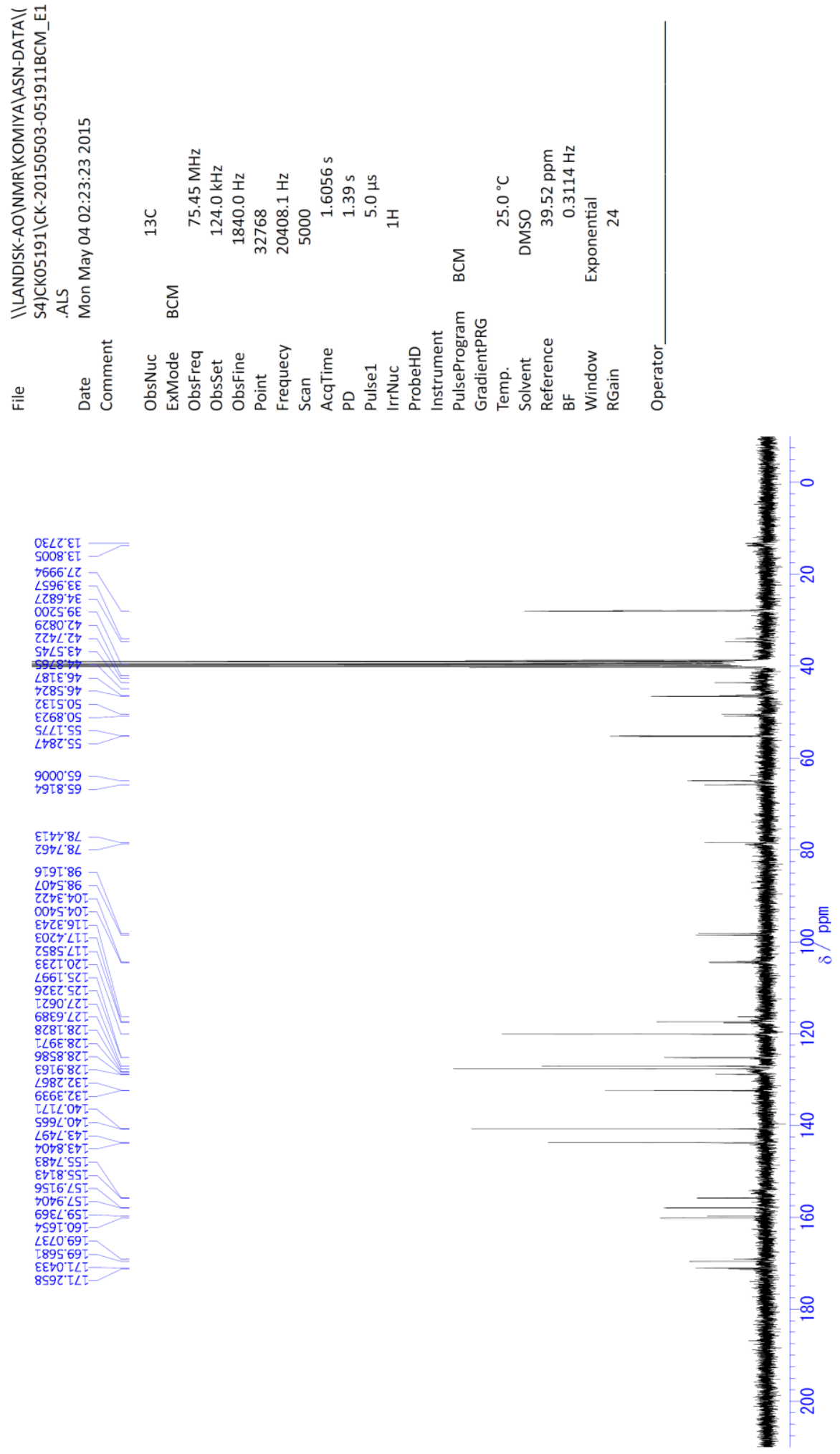
$\mathrm{N}^{2}-\left\{\left[(9 \mathrm{H}-\right.\right.$ Fluoren-9-yl)methoxy]carbonyl $\}-\mathrm{N}^{4}-\left\{2-\left[(\right.\right.$ tert-butoxycarbonyl)(ethyl)amino]ethyl $\}-\mathrm{N}^{4}$ (2,4-dimethoxybenzyl)- $L$-asparagine 29
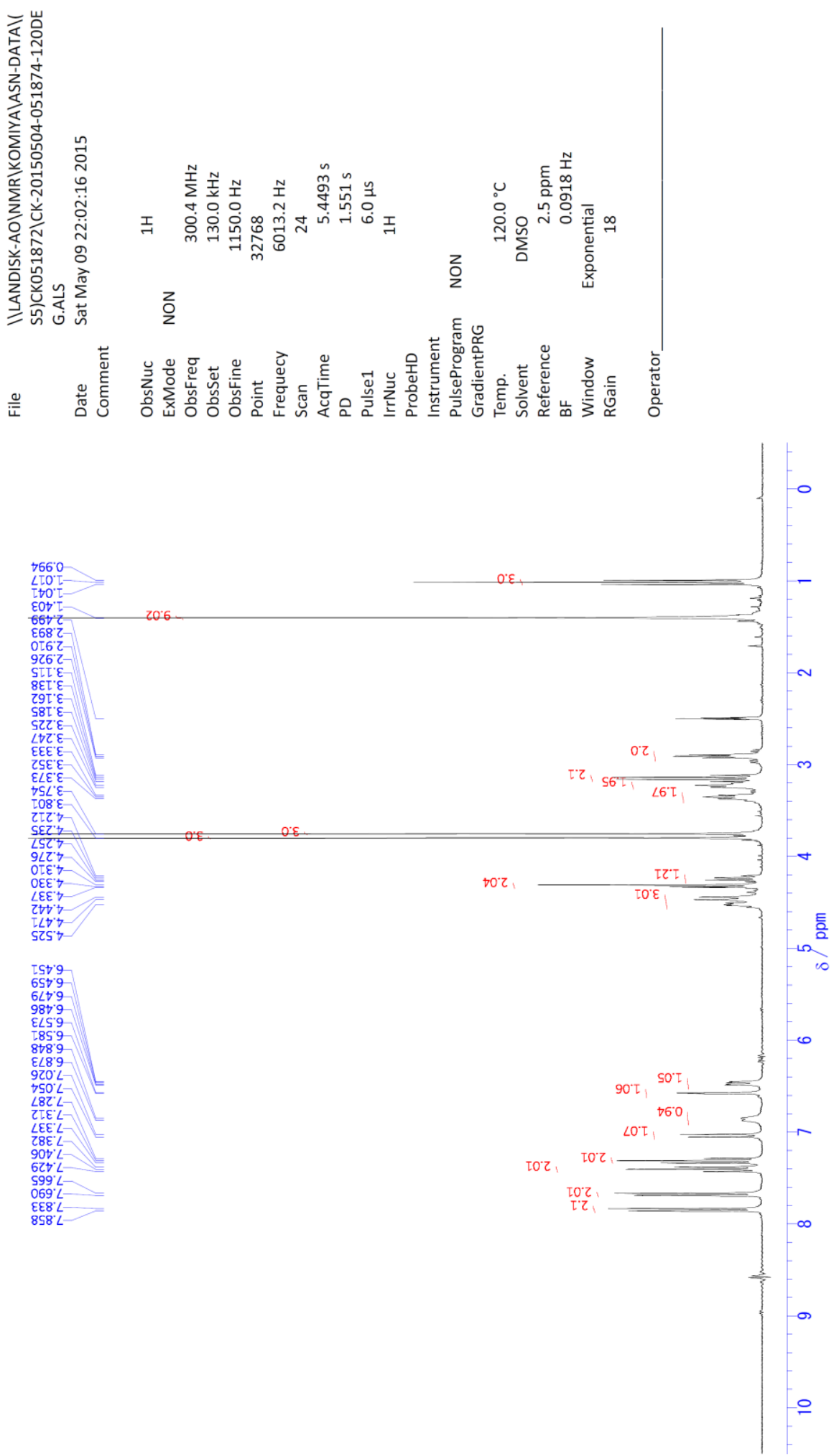

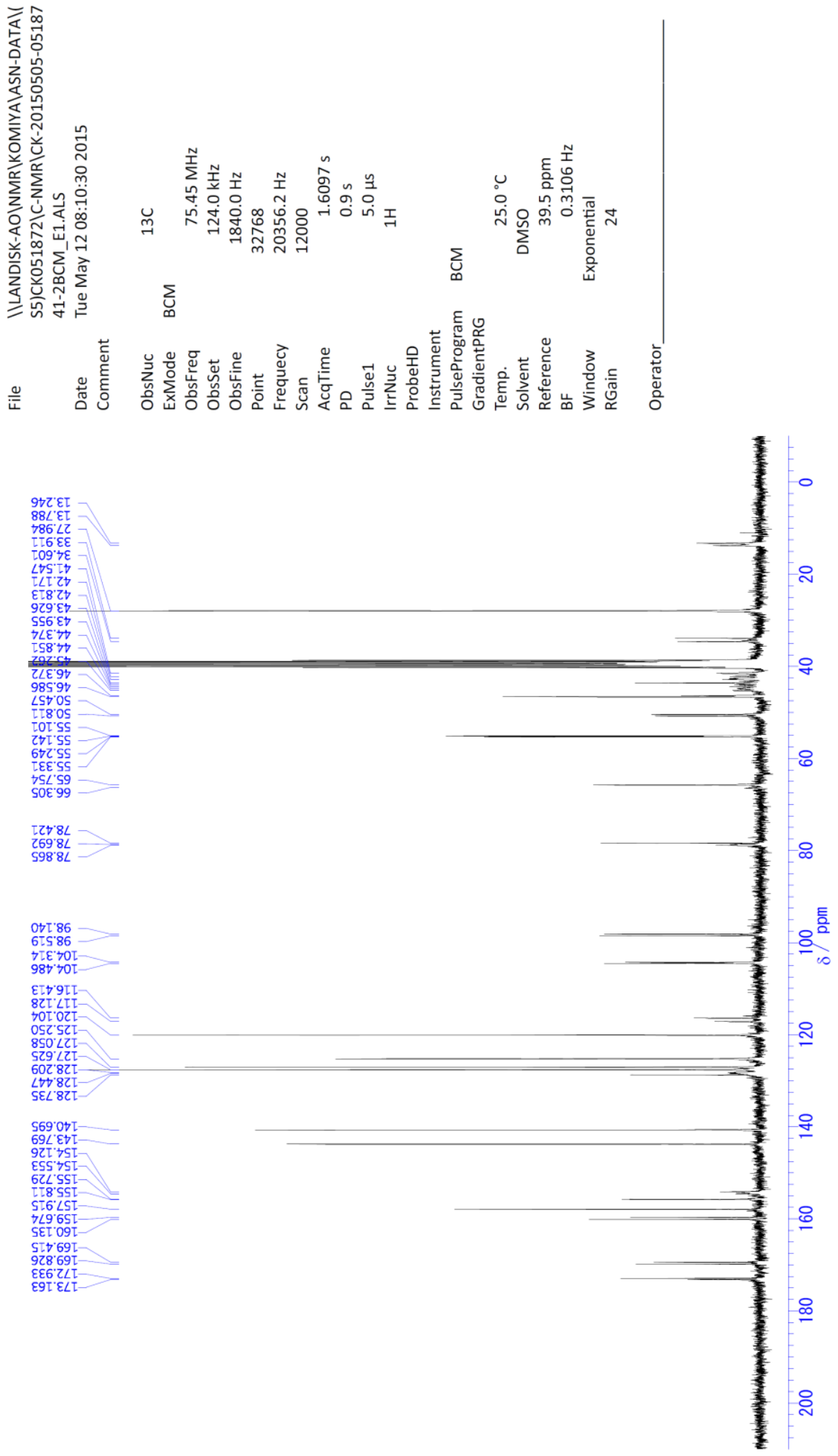